\title{
Self-Absorption and Luminescence Quantum Yields of Dye-Zeolite L Composites
}

\author{
André Devaux, ${ }^{*}{ }^{\dagger}$ Gion Calzaferri, ${ }^{\ddagger}$ Ivana Miletto, ${ }^{\dagger}$ Pengpeng Cao, ${ }^{\dagger}$ Peter Belser, ${ }^{\dagger}$ Dominik Brühwiler, $^{\S}$ \\ Oleg Khorev, ${ }^{\neq}$Robert Häner, ${ }^{\ddagger}$ and Andreas Kunzmann ${ }^{1}$ \\ ${ }^{\dagger}$ Department of Chemistry, University of Fribourg, Chemin du Musée 9, CH-1700 Fribourg, Switzerland \\ ${ }^{\ddagger}$ Department of Chemistry and Biochemistry, University of Bern, Freiestrasse 3, CH-3012 Bern, Switzerland \\ ${ }^{\S}$ Institute of Chemistry and Biological Chemistry, Zürich University of Applied Sciences, CH-8820 Wädenswil, Switzerland \\ ${ }^{\perp}$ Optical Additives GmbH, Flurweg 9, CH-3073 Gümligen, Switzerland
}

\begin{abstract}
Electronic absorption and fluorescence spectra based on transmission measurements of thin layers obtained from new perylene-zeolite L composites and new dye1,dye2-zeolite L sandwich composites, the latter acting as antenna systems, have been investigated and analyzed. The influence of extra- and intraparticle self-absorption on the spectral shape and fluorescence quantum yield is discussed in detail. Due to its intraparticle origin, self-absorption and re-emission can often not be avoided in organized systems such as dye-zeolite $\mathrm{L}$ composites where a high density of chromophores is a prerequisite for obtaining the desired photophysical properties. We show, however, that it can be avoided or at least minimized by preparing dye 1 , dye 2 -zeolite $\mathrm{L}$ sandwich composites where donors are present in a much larger amount than the acceptors because they act as antenna systems.
\end{abstract}

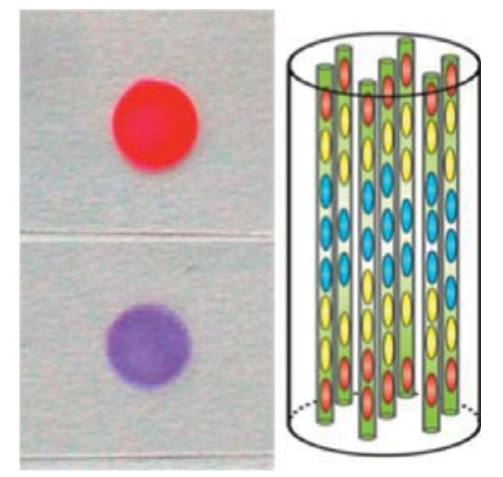

\section{INTRODUCTION}

Composites synthesized by embedding molecules, complexes, and clusters into the one-dimensional channels of zeolite $\mathrm{L}$ $(\mathrm{ZL})^{1,2}$ have recently experienced increasing interest from scientists engaged in different fields, ranging from very stable nontoxic pigments to novel optical materials with a large variety of different properties to promising objects for utilization in analytics, biology, diagnostics and drug delivery. This trend is exemplified by publications that appeared in $2013 .^{3-16}$ Electronic absorption and luminescence spectroscopy are important tools for characterizing the host-guest composites, and luminescence is often one of their key properties. Confocal luminescence microscopy on individual crystals has successfully been used and has contributed much to our current understanding of various dye-ZL properties. ${ }^{17-23}$ It is very often desirable to measure absorption and luminescence spectra of an ensemble in order to unambiguously interpret the observations and sometimes to derive luminescence quantum yields. Diffuse reflectance spectroscopy has frequently been used in the case for which this sophisticated instrumentation has been developed. ${ }^{10,24}$ Advancing dye-ZL research requires measurements that not only yield spectra and quantum yields as numbers but also provide information useful for understanding the mechanisms behind the observed phenomena. A prerequisite for these are high quality absorption and luminescence spectra where the band shapes can be interpreted unambiguously. This is best guaranteed if high quality transmission spectra are available. We have therefore successfully applied refractive index matching techniques in order to reduce or in favorable cases eliminate light scattering. We have used dispersions in liquid solvents or polymers as matrixes for dye-ZL composites, where sometimes a surface modification of the $\mathrm{ZL}$ crystals was required. ${ }^{25-27}$ More recently, a method for the preparation of thin oil-glass sandwiches (OGS) from dye-ZL composites has been employed. ${ }^{15,20}$ This technique has proven to be very convenient and allows differentiating between mechanisms that affect the shape of the absorption and luminescence spectra as well as the fluorescence quantum yields.

We have been mainly interested in medium sized, barrelshaped ZL crystals with average length and diameter of about $500 \mathrm{~nm}$ and in discs with a length and diameter of 200 and 800 $\mathrm{nm}$, respectively, as seen in the SEM images reported in Figure 1 (top part). The lower part of this figure (c, d, and e) illustrates the geometrical parameters of $\mathrm{ZL}$. A single channel of $\mathrm{ZL}$ is composed of unit cells with a length of $0.75 \mathrm{~nm} .^{28,29}$ From this, we deduce that a $500 \mathrm{~nm}$ long channel consist of 666 unit cells (uc), meaning that a maximum of 222 guest molecules with a length corresponding to 3 uc can occupy one channel. The number of parallel channels in such a crystal is impressive and perhaps larger than one might think at a first glance. It is approximately equal to $0.267\left(d_{\mathrm{Z}}\right)^{2}$ where $d_{\mathrm{Z}}$ is the diameter of the crystal in nanometers. A crystal with a diameter of $500 \mathrm{~nm}$ features nearly 67000 strictly parallel channels. 

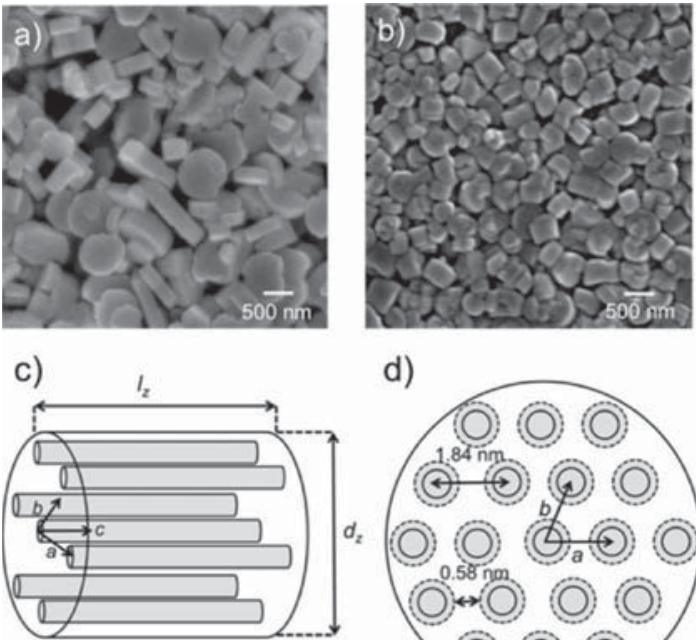

d)

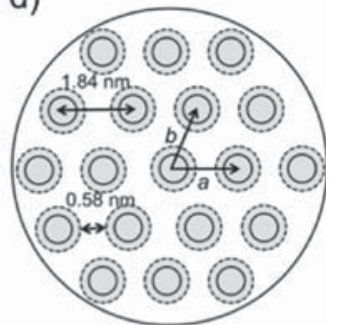

e)

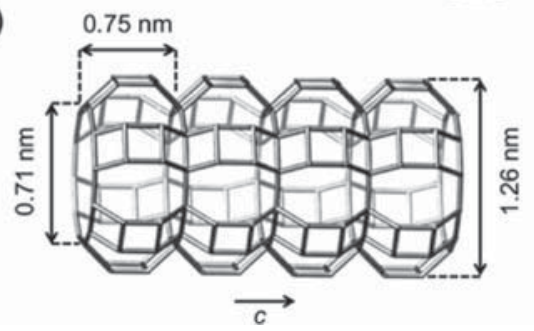

Figure 1. Morphology and geometrical parameters of zeolite L (ZL). (a) SEM image of disc-shaped ZL crystals with an average diameter of $500 \mathrm{~nm}$ and a thickness of $100 \mathrm{~nm}$. (b) Similar SEM image of barreltype ZL crystals with an average length and width of $500 \mathrm{~nm}$. (c) Representation of a single crystal as a cylinder with a system of $1 D$ parallel channels (depicted as gray tubes). Vectors $a, b$, and $c$ are primitive vectors, and $d_{\mathrm{Z}}$ and $l_{\mathrm{Z}}$ are the diameter and length of a crystal, respectively. (d) Schematic representation of the basal surface of ZL. The pore openings are shown as solid lines, and dashed lines represent the largest channel diameter. The center-to-center distance between two adjacent channels is $1.84 \mathrm{~nm}$ and the shortest distance is $0.58 \mathrm{~nm}$. (e) Side view of one ZL channel. The vector $c$ is the primitive vector coinciding with the channel axis. A unit cell is $0.75 \mathrm{~nm}$ long, the pore opening is $0.71 \mathrm{~nm}$ wide and the largest diameter in the channel is $1.26 \mathrm{~nm}$.

We describe the dye loading of a dye-ZL composite by means of the occupation probability $p$, as given by eq 1

$$
p=\frac{\text { number of occupied sites }}{\text { total amount of sites }}
$$

A site corresponds to the length that a dye occupies in terms of ZL unit cells ( $\mathrm{uc}$ ). The loading $p$ ranges from 0 for an empty ZL to 1 for a fully loaded one. The dye concentration $c(p)$ in a guest-ZL composite can be expressed with this as a function of the occupation probability, as described by eq $2^{1}$

$$
c(p)=0.752 \frac{p}{n_{\mathrm{s}}}\left(\frac{\mathrm{mol}}{\mathrm{L}}\right)
$$

where the symbol $n_{\mathrm{s}}$ expresses the number of uc occupied by a guest. It can, for example, be equal to 1,2 , or 3 , but must not necessarily be an integer. A number in the material name, such as dye-ZL.05 or dye-ZL.25, will indicate the loading of a dye-ZL composite. The composites given as examples above would, thus, have a loading $p$ of 0.05 and 0.25 , respectively. Figure 2 shows photographic images of four thin ZL layers on
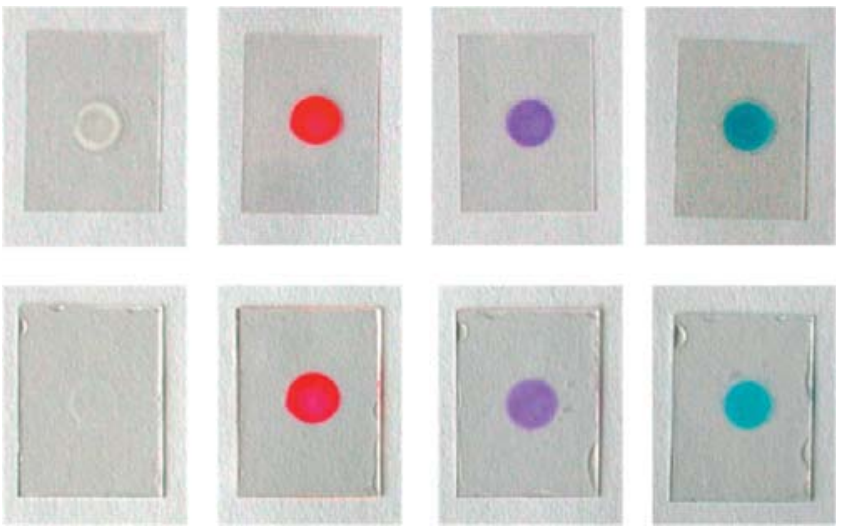

Figure 2. Photographic images of thin dye-ZL layers. The upper row are layers without addition of refractive index-matching oil, whereas the lower row shows the same samples after addition of such an oil and a second thin glass plate. From left to right: empty ZL, DXP-ZL.27, $\mathrm{Ox}^{+}-\mathrm{ZL} .02, \mathrm{Oxl}^{+}-\mathrm{ZL} .05$. The diameter of the sample spots is $0.8 \mathrm{~cm}$, and the amount of composite present in each spot is in the order of 0.2 mg.

glass in order to provide an impression of how the objects used in this study look. The leftmost layer was prepared from empty ZL crystals, whereas the other three contain dye-ZL composites (from left to right: DXP-ZL, $\mathrm{Ox}^{+}-\mathrm{ZL}$, and $\mathrm{Oxl}^{+}-\mathrm{ZL}$; the structural formulas of the dyes are given in Scheme 2). The samples in the upper row show more intense color because of strong light scattering, which is much reduced in the lower row as is probably best seen for the empty ZL layer on the left.

Differentiating interactions that can influence the spectroscopic properties of dye-ZL systems is a prerequisite for advancing our understanding of such materials. As in any classical situation, the reference systems must be highly diluted so that only interactions of the guest with the host, with the cosolvent, which may be present, and with the cocation can take place and, hence, be analyzed. This allows us to distinguish between new phenomena that arise from increased loadings and those that are caused by purely photophysical mechanisms such as self-absorption, Förster resonance energy transfer (FRET), or exciton coupling leading to, for example, Jaggregate bands. As an example, in a FRET situation, a low concentration of trap states can have a much higher importance than one might expect because these traps can be reached very efficiently. Adsorption of molecules at the outer surface of the crystals can sometimes dramatically influence the luminescence behavior of the dye-ZL composites. This has allowed the development of the stopcock principle, where molecules are attached specifically at the channel ends by means of van der Waals, Coulomb, or covalent interactions. Molecules that have been inserted into the channels of ZL can also leave them by the same route depending on the environmental conditions, which results in degradation of the composites. Plugging the channel entrances can prevent this. An impressive variety of stopcock modifications, used for different purposes, has been reported in the literature. ${ }^{9,30-38}$ We have applied this concept in the current study where necessary. It is also important to take into account the less specific adsorption of guests on the outer surface, where the tendency to form quenching aggregates can be remarkable. This fact has even been used to demonstrate the insertion kinetics of guests (e.g., see Figures 
16 and 17 in ref 1) but it has also been used for tuning other properties of the composites. ${ }^{12}$

An additional feature of dye-ZL composites is the sequential insertion of two different dyes 1 and 2, illustrated in Scheme 1.

Scheme 1. Representation of Two Different Organization Types in Dye 1,Dye2-ZL Composites Consisting of Dye Molecules Acting As Donors (Green) and Acceptors (Red)

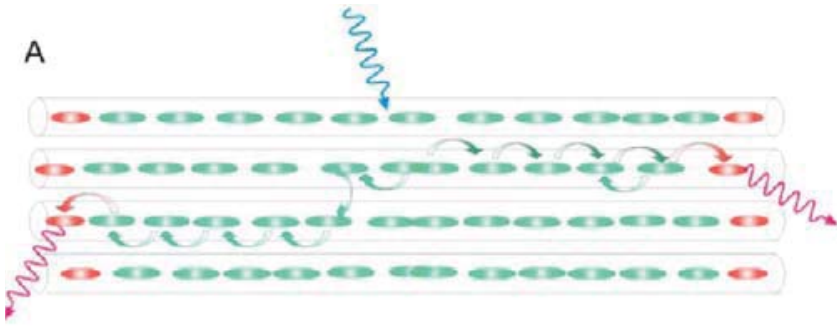

B

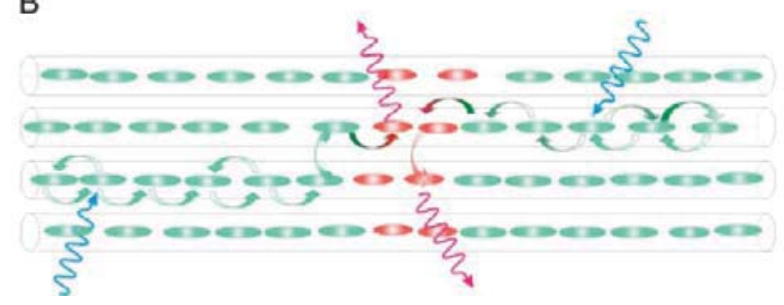

Sandwich-type, two-dye composites can be synthesized by this procedure, where one type of dye, absorbing light at shorter wavelength, is present in a large excess and acts as donor (green) for acceptors (red) present in much lower concentration. ${ }^{1,36,39}$ The following two types of organization will be discussed here: (A) acceptor,donor-ZL composites, where the donors are located in the middle part of the ZL channels and the acceptors at both ends, and (B) donor,acceptor-ZL composites, where the donors are at the entrances and the acceptors in the middle part of the channels. These composites exhibit antenna properties. Light is absorbed by the donor molecules and transported by means of FRET to the acceptors, which in arrangement $\mathrm{A}$ are located at both ends of the channels and in (B) are in the middle part of the channels. If efficient donor-to-acceptor transfer is desired, the acceptor should be reached before the donors have time to decay (e.g., by emitting light). This limits the acceptable channel length and is the reason why we have used in this study $\mathrm{ZL}$ crystals of the size indicated in Figure 1..$^{31}$

We first investigate the possibility of obtaining good quality transmission spectra of dye-ZL composites embedded in an oil-glass sandwich (OGS) by applying a light scattering baseline correction. This type of correction is especially necessary for samples containing small amounts of dyes, which we refer to as diluted materials. The absorption and fluorescence spectra of diluted dye-ZL composites are then compared with the spectra of the dyes in diluted DCM solutions. This allows monitoring the influence of the ZL host on the spectra. This measurement method forms the basis for investigating the influence of self-absorption on the shape of the spectral bands and on the fluorescence quantum yield. Furthermore, this method allows the differentiation between intra- and extraparticle processes and the comparison of properties of dye-ZL with dye1,dye2-ZL sandwich composites.

\section{EXPERIMENTAL SECTION}

Materials and Chemicals. The following chemicals were obtained from their respective suppliers and used without further purification: aluminum hydroxide $(\mathrm{ABCR}$, dried gel, $>99 \%$ ); Aerosil OX-50 (Degussa); Ludox HS-40 (40\% $\mathrm{SiO}_{2}$, Aldrich); potassium hydroxide (Fluka, purum p.a. $>85 \%$ ); sodium hydroxide (>98\%, Fluka); 1-butanol (Sigma-Aldrich, puriss p.a.); ethanol (Honeywell, p.a.); chloroform (Honeywell, p.a.); poly(methyl methacrylate) (Aldrich, $M_{\mathrm{w}}$ ca. 120000 ); immersion oil (Sigma, for microscopy); oxazine perchlorate (ABCR); base coat nail varnish (Lady Manhattan Cosmetics, Pro Shine Base Coat).

Oxazine118 nitrate was prepared following the synthesis procedure published in ref 40. N,N'-bis(4-tert-butyl-2,6dimethylphenyl)-3,4,9,10-perylenetetracarboxylic diimide (tbDXP), N,N'-bis[4-(2-oxopropoxy)-2,6-dimethylphenyl]3,4,9,10-perylenetetracarboxylic diimide (o-bone-DXP), and $N, N^{\prime}$-bis[4-(2-oxobutyl)-2,6-dimethylphenyl]-3,4,9,10-perylenetetracarboxylic diimide (bone-DXP) were synthesized according to newly developed procedures based on previously published works. ${ }^{41}$ A detailed description of these new procedures will be published in a separate paper. All absorption spectra were recorded with a Lambda 25 spectrophotometer (Perkin-Elmer) with a slit width of $1 \mathrm{~nm}$ and a scan speed of $120 \mathrm{~nm} / \mathrm{min}$. Luminescence spectra were obtained on an LS50B (Perkin-Elmer) by using a slit width of $7.5 \mathrm{~nm}$ for oilglass sandwiches and of $2.5 \mathrm{~nm}$ for liquid samples. The scan speed in both cases was $120 \mathrm{~nm} / \mathrm{min}$. Both spectrometers were equipped with a custom-built sample holder for the solid-state samples (see Figure 3). a)

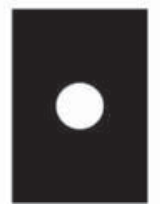

b)

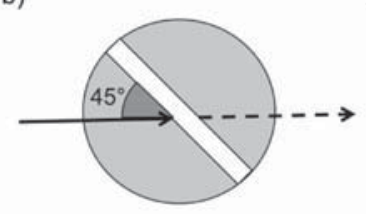

c)

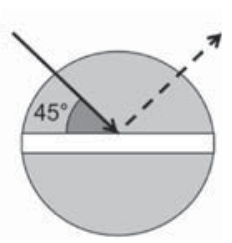

Figure 3. Working principle of custom-built sample holders used for the quantum yield determination of dye-ZL OGS. (a) Anodized black aluminum plate used as beam limiters. The OGS is placed between two such plates. (b) Sample holder used in the spectrophotometer. The gray disc represents the holder, the white bar is the slit into which the OGS-aluminum plate assembly is slid (held in place by Teflon screws), and the solid arrow indicates the incident light beam. (c) Sample holder used in the luminescence spectrometer (same legend as used for the previous holder).

The OGS is placed between two black anodized aluminum plates with the dye-ZL spot centered in the $6 \mathrm{~mm}$ hole. The whole assembly is then inserted into the sample holder slit and fixed by tightening two Teflon screws. The sample holder design ensures that the incident light beam hits the OGS at the same angle of $45^{\circ}$ in both the absorption and luminescence spectrometer, thus ensuring a similar geometry in both measurements, which is crucial for relative quantum yield determinations. The anodized black aluminum plates serve as beam limiters and help in avoiding reflection effects from the glass plates.

Zeolite L (ZL) Synthesis, Barrel- and Disc-Shaped. Barrel-shaped ZL crystals were synthesized following the procedure reported in ref 42 with an oxide ratio of 2.83 $\mathrm{K}_{2} \mathrm{O}: 1.00 \mathrm{Al}_{2} \mathrm{O}_{3}: 9.83 \mathrm{SiO}_{2}: 165.6 \mathrm{H}_{2} \mathrm{O}$. Usually, two $40 \mathrm{~mL}$ 
PTFE pressure vessels were used per synthesis run, and the two components for the synthesis gel were prepared as follows. An aluminate solution (solution A) was prepared by first dissolving $8.1 \mathrm{~g}$ of potassium hydroxide (Fluka, purum p.a. $\geq 85 \%$ ) in 25.4 $\mathrm{g}$ of doubly distilled water. Once the $\mathrm{KOH}$ was fully dissolved, $3.5 \mathrm{~g}$ of aluminum hydroxide (ABCR, dried gel, $\geq 99 \%$ ) was added and the solution refluxed for $16 \mathrm{~h}$. The slightly turbid solution was then cooled to rt and water loss was compensated. The silica solution (solution B) was prepared by adding $30.2 \mathrm{~g}$ of doubly distilled water to $12.9 \mathrm{~g}$ of Aerosil OX-50 (Degussa). The mixture was dispersed with an Ultra Turrax T18 basic (IKA) for $15 \mathrm{~min}$ at $16000 \mathrm{rpm}$. The dispersion was left standing at $\mathrm{rt}$ for $1 \mathrm{~h}$ before dispersing it again for $8 \mathrm{~min}$ at 16 $000 \mathrm{rpm}$.

After this second dispersion step, solution A was quickly poured into solution $\mathrm{B}$ under vigorous stirring. The resulting viscous white gel was aged for $3 \mathrm{~min}$ at $\mathrm{rt}$ under strong stirring before being evenly split into the two pressure vessels with a filling height corresponding to ca. $3 / 4$ of the vessel's total volume.

Crystallization took place in a rotating oven at $160{ }^{\circ} \mathrm{C}$ for 42 $\mathrm{h}$ with a rotation speed of $20 \mathrm{rpm}$. Once the reaction was finished, the vessels were cooled in an ice bath for $1 \mathrm{~h}$ before opening them. The milky white suspension was centrifuged for $15 \mathrm{~min}$ at $3100 \mathrm{rpm}$. The white residue was then washed with boiling doubly distilled water until the $\mathrm{pH}$ of the supernatant was 7 .

Disc-shaped ZL crystals were prepared following a procedure reported in ref 43. In a typical experiment, the aluminate component (solution A) was prepared by dissolving $9.32 \mathrm{~g}$ of $\mathrm{KOH}$ (Fluka, purum p.a. $\geq 85 \%$ ) and $5.87 \mathrm{~g}$ of $\mathrm{NaOH}(>98 \%$, Fluka) in $59.5 \mathrm{~g}$ of doubly distilled water. Aluminum hydroxide $(2.1 \mathrm{~g})$ was added once the hydroxides were fully dissolved. The reaction mixture was refluxed for $3 \mathrm{~h}$ in order to dissolve the aluminum hydroxide. The clear solution was cooled to rt and water loss was compensated. The silicate component (solution B) was prepared by mixing $58.9 \mathrm{~g}$ of Ludox HS-40 (40\% $\mathrm{SiO}_{2}$, Aldrich) with $4.8 \mathrm{~g}$ of ethanol (Honeywell, p.a.) and sonicating the mixture for $30 \mathrm{~min}$ at $\mathrm{rt}$.

Solution A was then poured quickly into solution B under strong stirring and the gel was aged for $10 \mathrm{~min}$ under stirring. The white gel was then evenly split on two PTFE pressure vessels ( $40 \mathrm{~mL}$ volume). Crystallization took place at $160{ }^{\circ} \mathrm{C}$ for $72 \mathrm{~h}$ in a rotating oven at a speed of $20 \mathrm{rpm}$. The workup procedure for the white product was similar to the one used for the barrel-type ZL.

Gas Phase Loading Procedure. In a typical experiment, $100 \mathrm{mg}$ of barrel-type zeolite L and $3.5 \mathrm{mg}$ of DXP $(p=0.5)$ were weighed into a $25 \mathrm{~mL}$ round-bottom flask. Dichloromethane (10 mL, technical grade) was then added, and the mixture was sonicated for $2 \mathrm{~min}$ to dissolve the dye and ensure a good dispersion of the zeolite. The solvent was then evaporated on a rotary evaporator $\left(40{ }^{\circ} \mathrm{C}, 600 \mathrm{mbar}\right)$, thus leading to a homogeneous coating of the zeolite surface with DXP. The coated zeolite was removed from the flask and transferred into an agate mortar, where it was ground into a fine powder. This powder was filled into a small glass ampule (25 $\mathrm{mm} \times 20 \mathrm{~mm}$ ) and dried on a vacuum line for $24 \mathrm{~h}$ at a pressure of $2 \times 10^{-2}$ mbar. The ampule was then sealed off under vacuum and put into a rotating oven or salt bath. The gas phase insertion process took place over $4 \mathrm{~d}$ at $260{ }^{\circ} \mathrm{C}$. Once this process was completed, the ampule was removed from the heating source and cooled to rt. The ampule was then opened, and the colored powder was washed three times with $20 \mathrm{~mL}$ portions of dichloromethane. The supernatant after the third washing was colorless. The effective loading degrees were determined by $\mathrm{HF}$ analysis.

Ion Exchange with $\mathrm{Ox}^{+}$and $\mathrm{Ox}^{+}$. The cationic dyes oxazine118 nitrate and oxazine1 perchlorate (ABCR) were incorporated into $\mathrm{ZL}$ by means of an ion-exchange process. In a typical experiment, $20 \mathrm{mg}$ of $\mathrm{ZL}$ was suspended in $2.2 \mathrm{~mL}$ of an aqueous solution of $\mathrm{Ox}^{+}$or $\mathrm{Oxl}^{+}$(corresponding to a target loading of $p=0.05$ ). The solution was then left stirring at $\mathrm{rt}$ for $18 \mathrm{~h}$. The dye-loaded ZL was then centrifuged off at $2900 \mathrm{rpm}$ for $15 \mathrm{~min}$ and washed two times with $20 \mathrm{~mL}$ portions of deionized water. The effective loading degrees were determined by HF analysis. Composite materials were usually prepared by first loading empty ZL with the donor perylene dye via gas phase adsorption followed by insertion of the cationic acceptor by ion exchange. In some cases, the dyes were inserted in the reverse order (i.e., first the cationic acceptor followed by the neutral donor).

Determination of Loading Level by HF Analysis. The effective loading levels were determined by first suspending 2 $\mathrm{mg}$ of the loaded material in $3 \mathrm{~mL}$ ethanol (Honeywell, p.a.) in a polystyrene cuvette. Six drops $(300 \mu \mathrm{L})$ of a $4 \%$ aqueous $\mathrm{HF}$ solution were added to dissolve the zeolite. The process was completed after $30 \mathrm{~min}$, leaving a transparent solution. The loading degree was then calculated from the dye concentration obtained from the UV-vis absorption spectrum.

Preparation of Oil-Glass Sandwiches or PMMA Thin Films for Spectroscopy. All absorption and luminescence spectra of dye-loaded ZL materials were recorded from oilglass sandwiches (OGS) prepared in a similar manner as is reported in refs 15 and 20. The samples were prepared by suspending $1 \mathrm{mg}$ of loaded material in $1 \mathrm{~mL}$ of 1-butanol (Sigma-Aldrich, puriss p.a.). A $200 \mu \mathrm{L}$ droplet was then deposited on a glass microscopy coverslip (Marienfeld, $24 \times 32$ $\mathrm{mm}$, no. 1). A steel ring with an inner diameter of $8 \mathrm{~mm}$, an outer diameter of $20 \mathrm{~mm}$, and equipped with an O-ring was used to confine the droplet's spread. Once the solvent was fully evaporated, the steel ring was removed and $300 \mu \mathrm{L}$ of immersion oil (Sigma, for microscopy) was deposited on the thin ZL layer. Placing a second coverslip on top of the immersion oil and sealing the sides with base-coat nail varnish (Lady Manhattan Cosmetics, Pro Shine Base Coat) completed the sandwich.

As an alternative to OGS, thin poly(methyl methacrylate) films containing dye-loaded ZL were prepared as follows. The dye-ZL composite $(1.1 \mathrm{mg})$ was suspended in $1 \mathrm{~mL}$ of chloroform. The homogeneous suspension was then poured into a glass Petri dish (35 mm diameter) containing $1 \mathrm{~mL}$ of a poly(methyl methacrylate) (Aldrich, $M_{\mathrm{W}}$ ca. 120000 ) solution in chloroform $(c=100 \mathrm{mg} / \mathrm{mL})$. The dish was then covered with a cardboard plate featuring a $6 \mathrm{~mm}$ hole, and the solvent was evaporated at rt. The solid transparent film was removed from the glass surface by soaking it in water for ca. $10 \mathrm{~min}$.

Relative Quantum Yield Measurements Using Thin Films. Fluorescence quantum yields $\Phi$ have been determined as relative values with respect to a standard with quantum yield $\Phi_{\mathrm{R}}$ according to eq 3, where the subscript R refers to the reference fluorophore of known quantum yield. In this equation, $I$ and $I_{\mathrm{R}}$ are the integrated intensity of the fluorescence band, expressed in wavenumbers, of the sample and the reference, respectively. $A$ and $A_{\mathrm{R}}$ are the absorptivity, which is the fraction of incident light which is absorbed by the 
Scheme 2. Structural Formulas of the Dyes Used in This Study $\left(\mathrm{Ox}^{+}=\right.$Oxazine 118; $\left.\mathrm{Ox}^{+}=\mathrm{Oxazine}^{+}\right)$

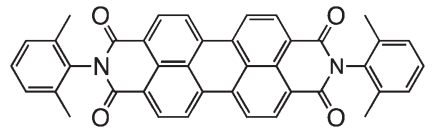

DXP

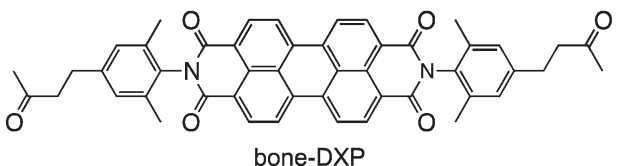

(N)

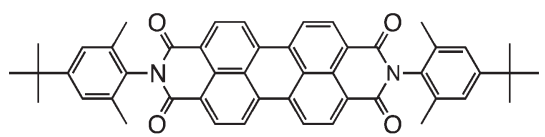

tb-DXP
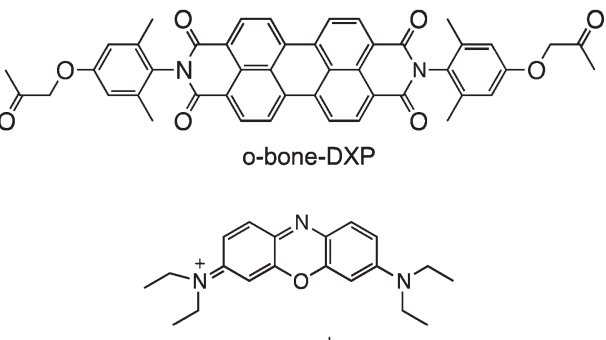

$\mathrm{O} \times 1^{+}$ sample, at identical excitation wavelength of the sample and the reference, respectively, and $n$ and $n_{\mathrm{R}}$ are the refractive indices of the corresponding media. Sample and reference have always been excited at the same wavelength, as assumed in expression 3 , so that it was not necessary to correct for the varying excitation intensities of different wavelengths.

$$
\Phi=\Phi_{\mathrm{R}} \frac{I}{I_{\mathrm{R}}} \frac{A_{\mathrm{R}}}{A}\left(\frac{n}{n_{\mathrm{R}}}\right)^{2}
$$

This is a standard procedure ${ }^{44}$ and has been used for determining the quantum yield of the perylene dyes in solution, using a value of $\Phi_{\mathrm{fl}}=0.98$ for DXP in degassed solution. ${ }^{41,45}$ Degassing was performed by applying the pump-freeze-thaw procedure three times. It is useful to consider error propagation more closely for determining relative luminescence quantum yields of thin layer OGS samples. We have always used and compared samples in an environment of identical refractive index, which means that the last term of eq 3 can be omitted. If we do not consider the error inherent to the quantum yield of the reference, which is reasonable because our focus is on relative yields in this study, the error propagation can be expressed as follows:

$$
\begin{aligned}
\Delta \Phi & =\frac{\Phi_{\mathrm{R}}}{I_{\mathrm{R}} A} \\
& {\left[A_{\mathrm{R}}^{2} \Delta I^{2}+\left(\frac{I}{I_{\mathrm{R}}} A_{\mathrm{R}}\right)^{2} \Delta I_{\mathrm{R}}^{2}+I^{2} \Delta I_{\mathrm{R}}^{2}+\left(I \frac{A_{\mathrm{R}}}{A}\right)^{2} \Delta A^{2}\right]^{1 / 2} }
\end{aligned}
$$

The most simple way to get a feeling of the consequences of this can be obtained by setting all values in this equation equal to one and assume an error of $5 \%$, which is a reasonable value, for each of them. The consequence of this is that the error $\Delta \Phi$ of the relative quantum yield $\Phi$ will be $10 \%$. It shows why it is so important to measure reference layers and sample layers under identical geometrical conditions both in absorptivity and fluorescence.

\section{RESULTS AND DISCUSSION}

The host-guest composites we investigated in this study have been prepared by loading the dyes depicted in Scheme 2. The absorption and fluorescence spectra of DXP, tb-DXP, boneDXP, and o-bone-DXP are identical, as illustrated in Figure 4. This is not surprising because they feature the same

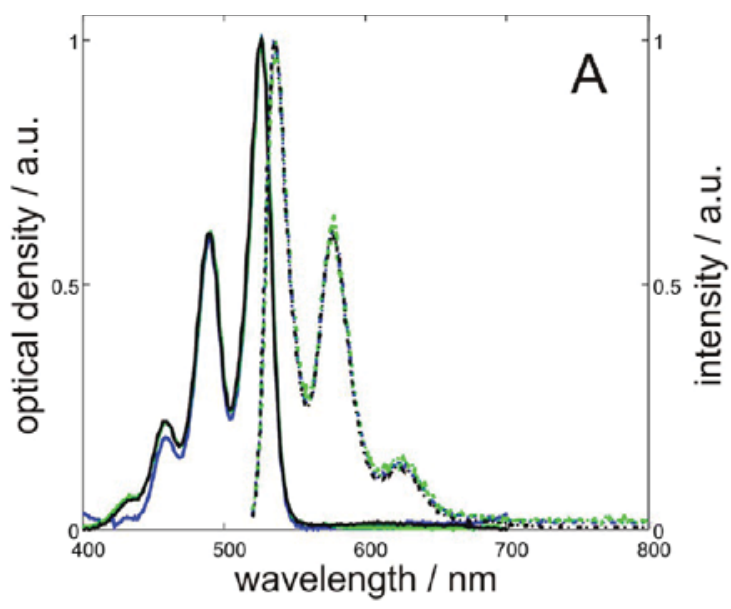

B

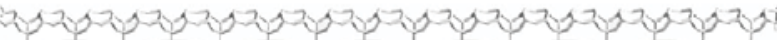

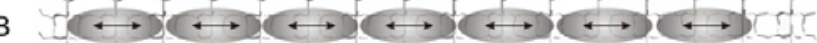

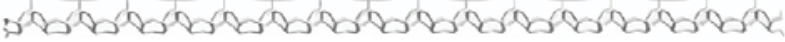

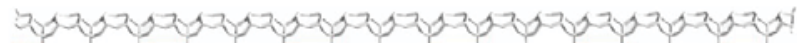

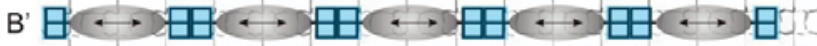

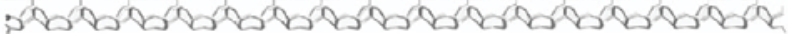

Figure 4. (A) Superposition of the absorption (solid) and fluorescence (dash-dot) spectra of DXP (red), tb-DXP (blue), bone-DXP (green), and o-bone-DXP (black) in about $10^{-7} \mathrm{M}$ degassed DCM solutions. All four spectra are perfectly overlapping. (B, $\left.\mathrm{B}^{\prime}\right)$ : Schematic explanation of the important difference between DXP (B) and the para-substituted tb-DXP, bone-DXP, and o-bone-DXP dyes $\left(\mathrm{B}^{\prime}\right)$ when densely packed inside of ZL channels. The substituent in the para position acts as spacers (blue rectangles).

chromophoric core. It is, however, important as it allows comparison of the properties of the corresponding peryleneZL composites (Table 1). The luminescence quantum yield of degassed, diluted solutions of these dyes in DCM is the same and close to one, within the experimental error, with the exception of the o-bone-DXP, which is about 10 times lower, in accordance with the observation of Langhals et al., who observed that a similar para-methoxy substituted dye shows a much lower quantum yield in a diluted solution than DXP. ${ }^{41}$ The reason for this dramatic fluorescence quenching is probably due to the interaction of the oxygen lone pairs via the phenyl ring with the perylene chromophore, leading to a partial quinoid resonance structure. An important difference between DXP and the para-substituted analogues is that the substituent in the para position acts as a spacer, increasing the 
Table 1. Photophysical Properties of the Dyes Used in This Study

\begin{tabular}{|c|c|c|c|c|}
\hline \multirow[b]{2}{*}{ dye } & \multicolumn{2}{|c|}{$\begin{array}{l}\text { absorption/emission } \\
\text { maxima }\end{array}$} & \multirow[b]{2}{*}{$\begin{array}{c}\text { extinction coefficient } \\
{\left[\mathrm{M}^{-1} \mathrm{~cm}^{-1}\right]}\end{array}$} & \multirow[b]{2}{*}{$\Phi_{\mathrm{fl}}$} \\
\hline & $\begin{array}{l}\text { in } \mathrm{ZL} \\
{[\mathrm{nm}]}\end{array}$ & $\begin{array}{l}\text { in solution } \\
{[\mathrm{nm}]}\end{array}$ & & \\
\hline \multirow[t]{2}{*}{ DXP } & $540 / 554$ & $525^{c} / 535^{c}$ & $88900^{a}$ & $0.98^{b}$ \\
\hline & & & $93300^{c}$ & \\
\hline tb-DXP & $540 / 554$ & $525^{c} / 535^{c}$ & $88200^{c}$ & 1.00 \\
\hline bone-DXP & $540 / 554$ & $525^{c} / 535^{c}$ & $85700^{c}$ & 0.96 \\
\hline $\begin{array}{l}\text { o-bone- } \\
\text { DXP }\end{array}$ & $540 / 554$ & $525^{c} / 535^{c}$ & $95100^{c}$ & 0.093 \\
\hline $\mathrm{Ox}^{+}$ & $596 / 607$ & $583^{d} / 599^{d}$ & $84100^{d}$ & $0.75^{d}$ \\
\hline $\mathrm{Ox}^{+}$ & $650 / 680$ & $640^{e} / 675^{e}$ & $121900^{e}$ & $0.11^{f}$ \\
\hline
\end{tabular}

minimum contact distance and, hence, decreasing the interaction leading to J-coupling (see eq 12 of ref 2 ) when the dyes are densely packed on the inside of the ZL channels. The carbonyl groups of the bone-DXP and o-bone-DXP substituents allow for an additional interaction and stabilization with the charge compensating potassium cations present in the channels, in a similar manner as reported for the fluorenoneZL and furo-furanone-ZL composites. ${ }^{46-48}$

Host-Guest Dye-ZL Composites Consisting of One Type of Dye As a Guest. We have already stated that a prerequisite for monitoring reliable luminescence quantum yields is that the absorption and luminescence spectra are measured under identical geometrical conditions and that the value of the absorptivity at the excitation wavelength can be determined with good accuracy. Fulfilling these criteria can be difficult for dispersions if sedimentation occurs during the measurement process, as is frequently observed for dye-zeolite composites where the particle size is in the order of several 100 $\mathrm{nm}$. We have therefore focused on thin layers, especially on the OGS technique and embedding into PMMA films, which are easier to handle and allow for much better reproducibility. If no surface modification is applied, zeolite crystals covered in an index-matching liquid or embedded in a polymer film of several $100 \mathrm{~nm}$ thickness always show some light scattering that increases at shorter wavelength. Although surface modification has been used for preparing different kind of composites, it is
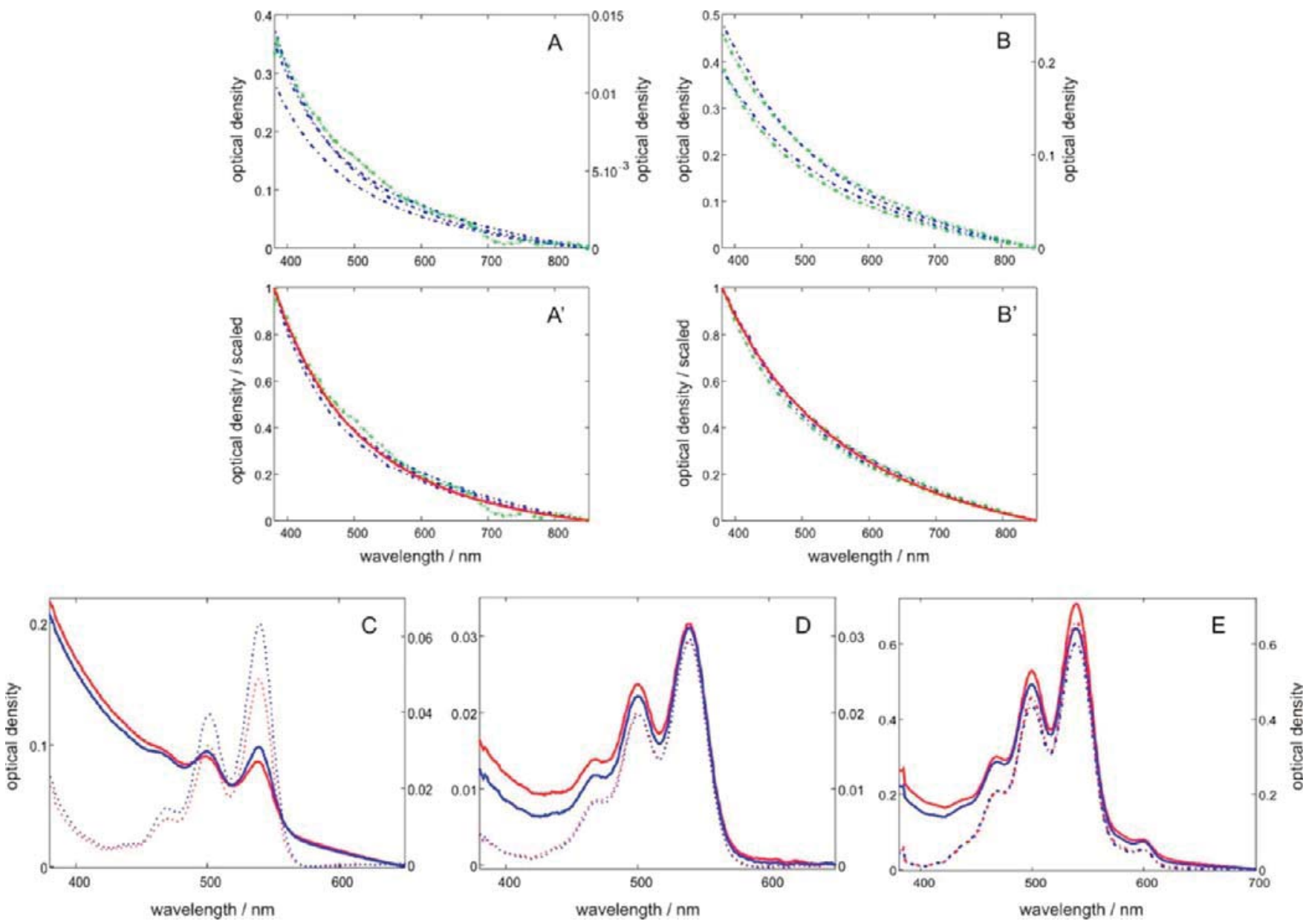

Figure 5. Baseline data of ZL and comparison of uncorrected and baseline corrected absorption spectra of different dye-ZL composites, measured in transmission as thin OGS layers on glass. Panels A and B show measurements of four different ZL OGS layers with different amounts of barreland disc-type ZL, respectively. The optical density scale on the left applies to the blue dash-dotted curves and to the green curve on the right. Panels $\mathrm{A}^{\prime}$ and $\mathrm{B}^{\prime}$ show absorption spectra scaled to the same height at $380 \mathrm{~nm}$ along with a comparison with eq 5 shown as a red curve, with exp $=3.0$ and 2.0 for the barrel- and for the disc-shaped zeolites, respectively. Panels $\mathrm{C}-\mathrm{E}$ show a comparison for two different layers (red and blue) of uncorrected (solid, left optical density scale) and baseline corrected (dashed, right optical density scale) absorption spectra: (C) DXP-ZL.01, (D) tb-DXPZL.05, (E) Ox ${ }^{+}$,tb-DXP-ZL.02,.3. 

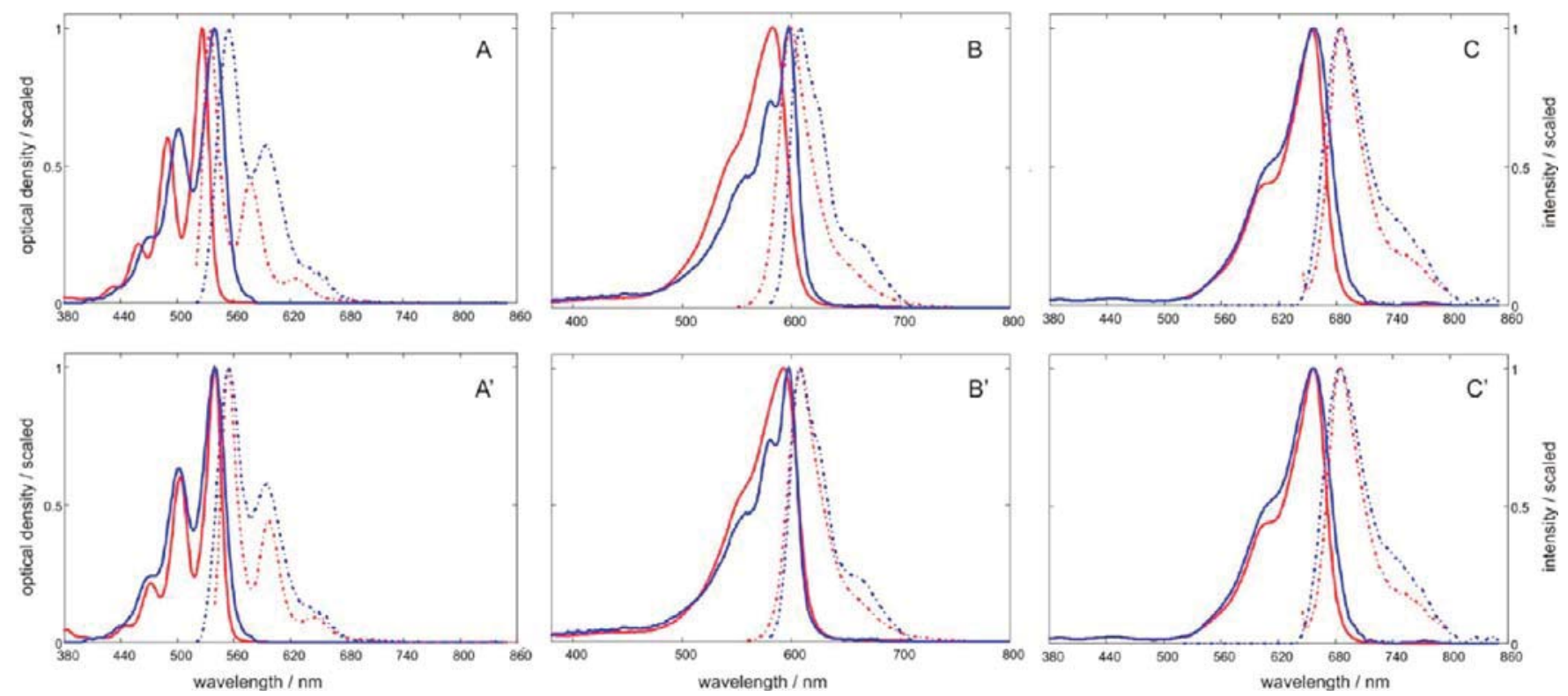

Figure 6. Comparison of the position and shape of the absorption (solid) and fluorescence (dash-dot) spectra of the dyes tb-DXP (A), Ox ${ }^{+}(B)$, and $\mathrm{Ox1}^{+}(\mathrm{C})$ in diluted DCM solutions (red) of about $10^{-7} \mathrm{M}$ and as OGS layers of dye-ZL.01 composites (blue) with an optical density of about 0.03 . Panels $\mathrm{A}^{\prime}-\mathrm{C}^{\prime}$ show the same spectra, but the solution spectra have been shifted so that the maxima coincide with those of the corresponding dyeZL spectra. The shifts in the maxima of both absorption and fluorescence bands are as follows: tb-DXP $\left(\mathrm{A}^{\prime}\right) 13 \mathrm{~nm}, 19 \mathrm{~nm} ; \mathrm{Ox}^{+}\left(\mathrm{B}^{\prime}\right) 10 \mathrm{~nm}, 9 \mathrm{~nm}$; $\left(C^{\prime}\right) 2 \mathrm{~nm}, 0 \mathrm{~nm}$. All spectra have been scaled to the same height at their respective maximum.

often not convenient for studying properties under many other conditions. ${ }^{25,26}$ An adequate baseline correction procedure for OGS layers of non-surface-modified dye-ZL composites would therefore be very useful. We found that this is actually possible in a convenient optical density range. Light scattering curves from barrel- and disc-shaped ZL crystals, as shown in the SEM images of Figure 1, as well as absorption spectra of dye-ZL composites (barrel type) are illustrated in Figure 5. We observe in the upper part of Figure 5 that the light scattering follows a smooth curve and that the Beer-Lambert law is fulfilled because measurements of layers prepared with different amounts of ZL coincide upon scaling to the same height, indicating that the crystals do not stick together and that the material is sufficiently homogeneous. Layers where crystals stick together to form larger particles cannot be used because of their chaotic scattering properties. The shape of the light scattering curve is different for the barrel type ZL, Figure 5(A, $\left.\mathrm{A}^{\prime}\right)$, and for the disc-shaped ones, Figure 5(B, $\left.\mathrm{B}^{\prime}\right)$. Interestingly, we observe that the shape of the light scattering curves can be wellrepresented as a function of the wavelength $\lambda$ following the empirical function shown in eq 5 , with the value of the exponent being 3 for the barrels and 2 for the discs. The last term enables termination of the correction at the longest wavelength $\lambda_{\max }$ of observation, which has the same purpose as setting the response of the spectrometer to zero at this wavelength. The fact that the exponents can in both cases be represented, within the experimental fluctuations, by integral numbers is very convenient for our purpose. However, this should be considered as being accidental, at least as far as we do not have a theoretical explanation for it. Different exponents are expected to be valid for different embedding environment and varying crystal shapes and sizes.

$$
\operatorname{shape}(\lambda)=\frac{1}{\lambda^{\exp }}-\frac{1}{\lambda_{\max }^{\exp }}
$$

The data for three representative dye-ZL composites given in the panels $\mathrm{C}, \mathrm{D}$, and $\mathrm{E}$ of Figure 5 illustrate that the baseline correction of the transmission spectra is very satisfactory and works well even for composites of very low loading and therefore weak dye response, where the signal of the scattering is larger than that of the dye. With this information we can now explore the influence of the zeolite host and the loading degree on the absorption and fluorescence spectra of the enclosed guests by comparing them to the spectra of dyes measured in diluted solutions.

In a $500 \mathrm{~nm}$ long barrel-shaped ZL crystal, we have at a loading of 0.01 on average about 2 molecules per channel, so that these composites can be understood as "ideally diluted" where interactions between dyes are negligibly small. The spectra of such composites are therefore well suited for comparison with those of dyes in highly diluted solutions. The result of such a comparison is illustrated in Figure 6 for three representative dyes, namely tb-DXP, $\mathrm{Ox}^{+}$, and $\mathrm{Oxl}^{+}$. The spectra are scaled to the same height at the corresponding maxima. A small bathochromic shift of a few nanometers is seen for the tb-DXP-ZL and the $\mathrm{Ox}^{+}-\mathrm{ZL}$ composites, but it is negligibly small for $\mathrm{Ox}^{+}-\mathrm{ZL}$. In order to better compare the shape, we have moved the position of the maxima of the solution spectra to match those of the dye-ZL as shown in Figure $6 A^{\prime}, B^{\prime}, C^{\prime}$. Interestingly, the vibrational features of the $\mathrm{Ox}^{+}-\mathrm{ZL}$ spectra are more distinct than those in solution. Such an observation has for the first time been reported for thionine-ZL composites and was interpreted as being due to the loss of conformational freedom of the dye inside of the channels, an interpretation that is supported by more recent studies. ${ }^{15,50,51}$ More importantly we can conclude that the shape of the absorption spectra of these dyes is not affected by interactions with the host, apart from these minor wellunderstood features. This means that if changes are observed at higher loading, they must be due to another mechanism. The first of the mechanisms that we will discuss now is self- 

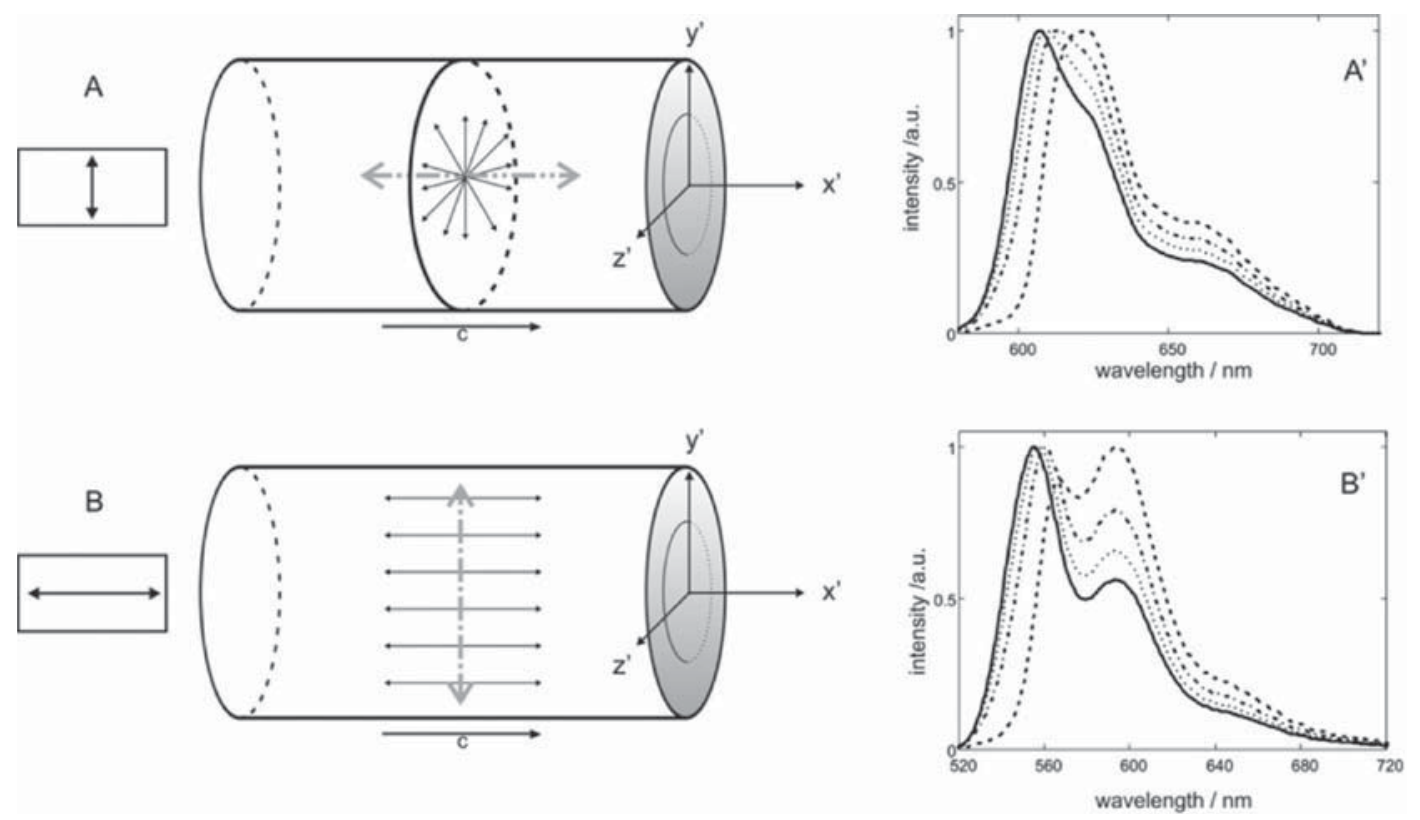

Figure 7. Model calculations of the influence of intrazeolite self-absorption on the shape of fluorescence spectra. The gray dash-dotted double arrows indicate the direction of light absorption and emission. (A) The orientation of the ETDM of the $\mathrm{Ox}^{+}$molecule in $\mathrm{Ox}^{+}-\mathrm{ZL}$ is perpendicular to the $c$ axis of the ZL channel (double arrows, solid). (B) The molecule DXP and its ETDM are oriented parallel to the $c$ axis of the ZL channel axis in the DXP-ZL composite (double arrows, solid). ( $\mathrm{A}^{\prime}$ and $\mathrm{B}^{\prime}$ ) Fluorescence spectra of $\mathrm{Ox}^{+}-\mathrm{ZL}$ and of DXP-ZL, respectively. $p=0.01$ (measured), solid; $p=0.1$, dotted; $p=0.25$, dash-dot; $p=0.75$, dashed (all spectra of higher loaded materials are calculated). The spectra are scaled to the respective maximum.

absorption, a process that can be easily underestimated in such composites, as has been pointed out previously and that we now explore in more detail. ${ }^{15,27}$

The change induced by self-absorption, or inner filter effect, on the shape and intensity of luminescence spectra at high optical density is well-known. It is caused by reabsorption of the short wavelength photons emitted from a fluorophore by neighboring molecules of the same type, resulting in a red shift of the observed emission. Attenuation of the blue emission edge is most pronounced for fluorophores showing significant overlap between their absorption and the emission bands. Fluorophores that display a large Stokes shift are less sensitive to this phenomenon. ${ }^{44,52}$ Although self-absorption and reemission can be circumvented for ordinary solutions by diluting the sample and monitoring the path length, it cannot always be avoided in organized systems such as the dye-ZL composites investigated here, where a high density of chromophores is a prerequisite for obtaining the desired photophysical properties. It is therefore necessary to discuss this phenomenon in some detail. The influence of intracomposite self-absorption may easily be underestimated if the particle size of the dye-ZL composites investigated is in the order of a few hundred $\mathrm{nm}$. This can best be demonstrated by performing model calculations. We did this on dye-ZL composites in a similar way as explained in ref 53 on the basis of reasoning reported previously, by assuming ideal behavior of the dyes meaning no interactions such as those leading to, for example, J-coupling and by assuming no internal reflection. ${ }^{52,54}$ The latter part indicates that the composites are embedded in a perfect refractive index-matching support. This allows a simulation exclusively of the influence of self-absorption on the shape of the fluorescence band. Results obtained for ZL hosts with barrel-shaped morphology that have an equal diameter and length of $500 \mathrm{~nm}$ and contain two different, noninteracting chromophores are illustrated in Figure 7. In the case of the $\mathrm{Ox}^{+}-\mathrm{ZL}$ composite, the dye's electronic transition dipole moment (ETDM) is oriented perpendicular to the $c$ axis. ${ }^{18,19,51}$ In the other case, a DXP-ZL composite, the ETDM is aligned with the $c$ axis of the zeolite crystal. ${ }^{21}$ All spectra are scaled to unity at their respective maximum for better comparison. We observe that the longer wavelength band intensities increase at the expense of the short wavelength part faster than one might have expected for a $500 \mathrm{~nm}$ particle. This behavior is due not only to the large concentration of chromophores made possible by the ZL host but also to the pronounced optical anisotropy of the composites. The most elegant way to circumvent this selfabsorption mechanism is by synthesizing antenna materials, containing a large concentration of donors with only a few acceptor molecules, as depicted in Scheme 1. ${ }^{1}$ Such systems will be discussed later. We should add that modeling of the long wavelength tail is delicate because it strongly depends on the quality of the experimental spectrum, which has to be measured for dye-ZL samples of very low loading, as is the case for the solid line in Figure $7 \mathrm{~A}^{\prime}, \mathrm{B}^{\prime}$. It is naturally noisier and therefore the intensity is underestimated for the spectra shown in Figure 7. This does not, however, affect the main conclusions gained. A superposition of intra- and extraparticle self-absorption will generally be observed in experimental spectra of thin layers. We show in Figure $8 \mathrm{~A}-\mathrm{C}$ spectra of samples where no distinction between intra- and extrazeolite self-absorption is made. A clear distinction can be made in the cases of Figure $8 \mathrm{~A} 1-\mathrm{C} 1$, where we only show the fluorescence spectra because no difference in the absorption bands was observed. The spectra are not scaled in Figure 8A, A1 but are scaled in all other cases. The absorption spectra in Figure 8A show little change of their shape, with the exception of first signs of saturation in the case of the tb-DXP-ZL.27 composite. However, important changes occur in the fluorescence band shape so that the fluorescence 

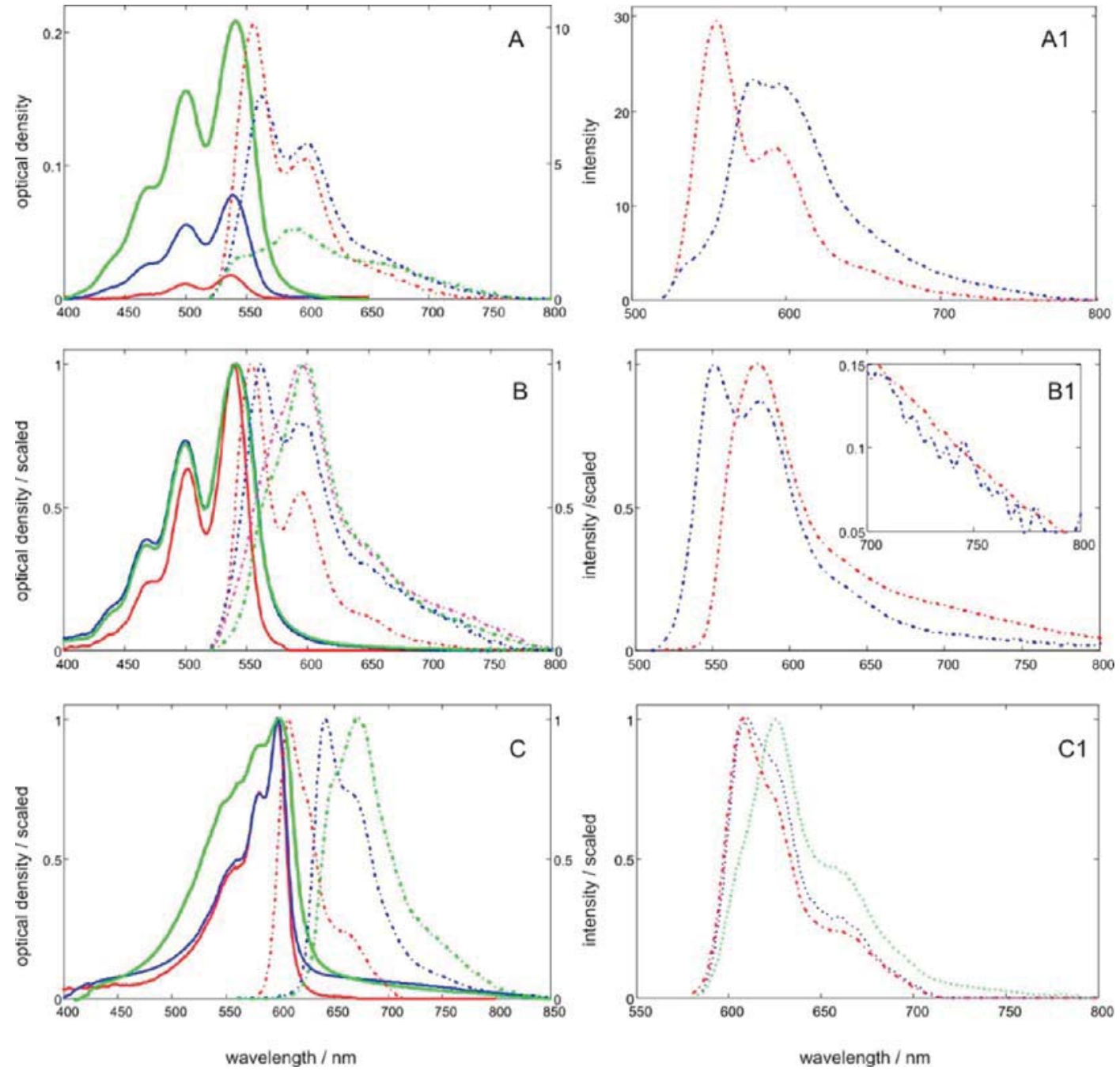

Figure 8. Experimental observations of the influence of intra- and extrazeolite self-absorption on the shape of fluorescence spectra. Absorption spectra are presented as solid and luminescence spectra as dash-dotted lines. We show in the panels $\mathrm{A}-\mathrm{C}$ absorption as well as fluorescence spectra with no distinction between intra- and extrazeolite self-absorption, and this distinction is shown in panels $\mathrm{A} 1-\mathrm{C} 1$. The spectra $\mathrm{B}$ and $\mathrm{B} 1$ as well as $\mathrm{C}$ and $\mathrm{C} 1$ are scaled to the same height at the corresponding maxima. (A) tb-DXP-ZL.01, red; tb-DXP-ZL.05, blue; and tb-DXP-ZL.27, green. (A1) tb-DXP-ZL.01, red; tb-DXP-ZL.05, blue. (B) DXP-ZL.01, red; DXP-ZL.35a, blue; DXP-ZL.35b, pink; DXP-ZL.35c, green. (B1) DXPZL.35A dense layer, red; DXP-ZL.35B diluted by a factor of 7.8, blue. (C) Ox ${ }^{+}-\mathrm{ZL} .01$, red ; $\mathrm{Ox}^{+}-\mathrm{ZL} .05$ large concentration, blue, Ox ${ }^{+}-\mathrm{ZL} .10 \mathrm{c}$, green. (C1) Ox ${ }^{+}-\mathrm{ZL} .02$, red; thicker layer 1, blue; thicker layer 2, green. All sample details are reported in Table 2

band of the tb-DXP-ZL.27 material could hardly be identified to originate from tb-DXP if we did not understand its origin. At the same time, the fluorescence intensity decreases; it is strongest for the tb-DXP-ZL.01, despite its low absorptivity of 0.04 (see Table 2). The fluorescence intensity drops to $20 \%$ for the tb-DXP-ZL.05 layer with an absorptivity of 0.164 and becomes very weak for tb-DXP-ZL.27. Interestingly, the fluorescence quantum yield of the tb-DXP-ZL.05 sample increases very much for layers with lower absorptivity. We show in Figure 8A1 that a layer with an absorptivity of 0.073 that exhibits a fluorescence quantum yield of $83 \%$. Extraparticle selfabsorption is responsible for the important difference in band shape and intensities between the tb-DXP-ZL.01 sample and the concentrated tb-DXP.05 layer. The emission spectrum recorded from the tb-DXP-ZL.01 layer shows the undistorted fluorescence band as expected of the tb-DXP molecule inside of the ZL channels. In the case of tb-DXP-ZL.05 material, measuring layers containing fewer composites can restore this undistorted spectrum.
This is in agreement with the modeling illustrated in Figure $7 \mathrm{~B}$, which shows that the influence of intraparticle selfabsorption at these loadings is small. The origin of the spectral changes seen in Figure 8B is more complex. Two changes are seen in the samples with a loading of $p=0.35$ with respect to the spectra of DXP-ZL.01, $A=0.115$ in red. The three emission spectra of DXP-ZL.35 were recoded from the same material but with layers of increasing particle concentration and, hence, increasing absorptivity; Table 2 . The shape of the absorption spectra of the three layers labeled by the letters $a, b$, and $c$ are identical but exhibit a new long wavelength shoulder in the region of 550 to $620 \mathrm{~nm}$. The increasing influence of selfabsorption with increasing absorptivity is very pronounced and accompanied by a decrease of the fluorescence quantum yield. Apart from this we see a very similar long wavelength tail above $700 \mathrm{~nm}$ in all three spectra. Furthermore, the spectral behavior does not correspond to the development expected from selfabsorption. This feature and the tail in the absorption spectra of the DXP-ZL.35 originate from J-coupling as has been recently 
Table 2. Fluorescence Quantum Yields ${ }^{a}$

\begin{tabular}{|c|c|c|c|c|c|c|c|}
\hline sample & $\lambda_{\mathrm{ex}}$ & $A$ & $\Phi_{\mathrm{fl}}$ & sample & $\lambda_{\mathrm{ex}}$ & $A$ & $\Phi_{\mathrm{fl}}$ \\
\hline tb-DXP-ZL.01; A & 490 & 0.04 & 1.00 & $\mathrm{Ox}^{+}-\mathrm{ZL} .001^{c}$ & & & $\sim 1^{c}$ \\
\hline tb-DXP-ZL.05; A & 490 & 0.164 & 0.20 & $\mathrm{Ox}^{+}-\mathrm{ZL} .02 ; \mathrm{C}$ & 530 & 0.32 & 0.75 \\
\hline tb-DXP-ZL.27; A & 490 & 0.381 & 0.04 & $\mathrm{Ox}^{+}-\mathrm{ZL} .05 ; \mathrm{C}$ & 540 & 0.85 & 0.38 \\
\hline tb-DXP-ZL.01; A1 & 490 & 0.045 & $1^{b}$ & $\mathrm{Ox}^{+}-\mathrm{ZL} .10 ; \mathrm{C}$ & 530 & $\sim 1$ sat & 0.1 \\
\hline tb-DXP-ZL.05; A1 & 490 & 0.073 & 0.83 & $\mathrm{Ox}^{+}-\mathrm{ZL} .02 \mathrm{~A} ; \mathrm{C} 1$ & 540 & 0.14 & 0.41 \\
\hline DXP-ZL.01; B & 490 & 0.115 & 0.8 & $\mathrm{Ox}^{+}-\mathrm{ZL} .02 \mathrm{~B} ; \mathrm{C} 1$ & 540 & 0.16 & 0.35 \\
\hline DXP-ZL.35a; B & 490 & 0.20 & 0.2 & $\mathrm{Ox}^{+}-\mathrm{ZL} .02 \mathrm{C} ; \mathrm{C} 1$ & 540 & 0.32 & 0.75 \\
\hline DXP-ZL.35b; B & 490 & 0.50 & 0.12 & & & & \\
\hline DXP-ZL.35c; B & 490 & 0.90 & 0.07 & Ox1,DXP-ZL.3,.02a; ns & & 0.085 & 0.3 \\
\hline DXP-ZL.35A; B1 & 490 & 0.46 & 0.16 & Ox1,DXP-ZL.3,.02b; ns & & 0.32 & 0.11 \\
\hline DXP-ZL.35B; B1 & 490 & 0.075 & 0.2 & Ox1,bone-DXP-ZL.3,.02A ${ }^{d}$ & & 0.072 & 0.26 \\
\hline DXP-ZL.5a; ns & 490 & 0.90 & 0.065 & Ox1,bone-DXP-ZL.3,.02B ${ }^{d}$ & & 0.11 & 0.06 \\
\hline DXP-ZL.5b; ns & 490 & 0.52 & 0.1 & & & & \\
\hline DXP-ZL.5c; ns & 490 & 0.18 & 0.13 & tb-DXP,Ox-ZL.05,. $3^{e}$ & 490 & 0.17 & 0.7 \\
\hline
\end{tabular}

${ }^{a} \lambda_{\text {ex }}=$ excitation wavelength $[\mathrm{nm}] ; A=$ absorptivity at maximum; $\Phi_{\mathrm{fl}}=$ fluorescence quantum yield. The letters $\mathrm{A}, \mathrm{Al}, \mathrm{B}$, and so forth after the name of the samples refer to the corresponding spectra seen in Figure 8. ns = spectra not shown; sat = saturated spectrum. ${ }^{b}$ Ref $21 .{ }^{c}$ Ref $55 .{ }^{d}$ See Figure $9 \mathrm{~A}$ and $\mathrm{A}^{\prime} .{ }^{e}$ See Figure $9 \mathrm{~B}$ and $\mathrm{B}^{\prime}$.

demonstrated and discussed in detail. ${ }^{21}$ This coupling has so far not been observed in tb-DXP-ZL, bone-DXP-ZL, or o-boneDXP-ZL, which allows us to conclude that the substituents of theses dyes keep them at a sufficient distance so that these interactions are too small to be noticed. The J-coupling interaction decreases following an inverse distance power to the third relation, as discussed, for example, in eq 9.47 of ref 36 . Figure 8B1 shows layers made from the same DXP-ZL.35 composite material but with two different layer thicknesses: the blue spectrum has been recorded from a layer that was diluted by a factor of 7.8 with respect to the red one. Further dilution did not lead to any significant changes in the spectral shape. This means that the distortions seen in the blue spectrum results from intraparticle self-absorption. As discussed above, Jcoupling leads to a tail at longer wavelengths. The inset shows that its shape is independent of dilution. The quantum yield increases only moderately with dilution, indicating that the quenching is caused by the intra-ZL self-absorption mechanism. The $\mathrm{Ox}^{+}-\mathrm{ZL}$ samples exhibit a similar behavior (Figure $8 \mathrm{C}, \mathrm{C} 1)$. We have added a spectrum of an optically dense $\mathrm{Ox}^{+}-$ ZL.10 layer, with absorptivity close to one, where the absorption spectrum shows remarkable optical saturation. It would not be possible to trace the shape of the $\mathrm{Ox}^{+}-\mathrm{ZL} .05$ and $\mathrm{Ox}^{+}-\mathrm{ZL} .10 \mathrm{c}$ fluorescence spectra back to $\mathrm{Ox}^{+}$fluorescence without the necessary information provided by the dilution series of the Ox ${ }^{+}-$ZL.02 layers shown on the right side of Figure 8 . The fluorescence quantum yields of the $\mathrm{Ox}^{+}-\mathrm{ZL}$ composites appear to be a bit strange. We show these values to demonstrate that $\mathrm{Ox}^{+}-\mathrm{ZL}$ and, even more, $\mathrm{Ox} 1^{+}-\mathrm{ZL}$ composites are quite difficult to handle because the dyes have a stronger tendency to diffuse out of the channels and to form quenching aggregates at the outer surface. Fluorescence quenching is weak in the case of the $\mathrm{Ox}^{+}-\mathrm{ZL} .02 \mathrm{C}$; $\mathrm{C} 1$ layer. However, stable $\mathrm{Ox}^{+}-\mathrm{ZL}$ and $\mathrm{Oxl}^{+}-\mathrm{ZL}$ can only be obtained by proper sealing of the channel entrances so that such diffusion processes cannot occur. The same is true for the perylene-ZL composites, but they are kinetically more robust and therefore easier to handle in such experiments.

The examples we have discussed so far demonstrate the importance of understanding the reason for changes seen in the absorption and fluorescence spectra of dye-ZL composites in order to avoid reaching wrong conclusions regarding photo- physical/photochemical mechanisms. They also illustrate, together with Figure 6, the importance of using composites with low loading and sufficiently low optical density as a reference. Also, the fluorescence quantum yield of all dye-ZL samples discussed, with the exception of the o-bone-DXP, are large and close to one for samples of low loading. Some traps are always present and quench accordingly in each selfabsorption step and reduce the re-emitted light. Scattering causes further losses after each self-absorption and re-emission step. We do not try to systematize these observations further. The important message is that the richness of observations is in no case caused by host-guest interactions but is due to the essentially simple optical self-absorption mechanism, to guestguest interactions inside of the channels, or to quenchers on the outer surface of the composites (e.g. created by guests diffusing out of the channels and forming aggregates at the outer surface or guests that remained at the outer surface after the preparation procedure). There are other cases where interactions of the guest with the host, with cosolvents present and with cocations, are important and sometimes are the properties-determining parameters. We have discussed some of this in refs 55,56 and do not intend to further explore this here, where we put the emphasis on the important consequences of the aspects we have discussed above.

Host Guest Dye-ZL Composites Consisting of Two Dyes, Forming a Sandwich Organization. We extend the systems reported and discussed in the previous section to sandwich structures as explained in Scheme 1. We will begin with the case where donor molecules are first inserted into the channels and then followed by a few acceptors according to the scheme shown in Figure 12 of ref 1 . This leads to what we call an acceptor,donor-ZL composite. We chose composites where first one of the perylene dyes in Scheme 2 has been inserted from the gas phase, followed by $\mathrm{Ox}^{+}$addition from solution by means of ion exchange. The length and shape of perylene and $\mathrm{Oxl}^{+}$are such that the ETDM of the $\mathrm{S}_{0}-\mathrm{S}_{1}$ transitions are both oriented parallel to the channel axis. ${ }^{36}$ This results in large Förster radii for both homo-FRET in the perylene domain and FRET from the donor perylene to the acceptor $\mathrm{Oxl}^{+}$. The Förster radius indicates the distance at which the probability of a FRET process taking place is equal to $50 \% .^{36}$ The calculated radii are reported in Table 3. A complication arises in this 
Table 3. Homo and Donor-Acceptor FRET Data

\begin{tabular}{lccl} 
chromophore & $\begin{array}{c}\text { oscillator } \\
\text { strength }\end{array}$ & $\begin{array}{c}\text { spectral overlap }\left[\mathrm{L} \mathrm{cm}^{3} /\right. \\
\text { mol }]\end{array}$ & $\begin{array}{c}\text { Förster radius } \\
{[\mathrm{nm}]}\end{array}$ \\
tb-DXP & 0.736 & $1.75 \times 10^{-13}$ & $7.2\left(\kappa^{2}=4\right)$ \\
$\mathrm{Ox}^{+}$ & 0.70 & $3.0 \times 10^{-13}$ & $6.2\left(\kappa^{2}=1\right)$ \\
$\mathrm{Oxl}^{+}$ & 0.92 & $2.95 \times 10^{-13}$ & $7.85\left(\kappa^{2}=4\right)$ \\
tb-DXP- $^{+}{ }^{+}$ & & $4.18 \times 10^{-13}$ & $6.6\left(\kappa^{2}=1\right)$ \\
tb-DXP- $^{\text {Oxl }}{ }^{+}$ & & $4.3 \times 10^{-13}$ & $8.36\left(\kappa^{2}=4\right)$ \\
& & & \\
\hline
\end{tabular}

system from the fact that $\mathrm{OxI}^{+}$has the tendency to leak out of the channels under most conditions within a couple of hours if the channels are not plugged quickly after preparation. We have already stated that especially cationic molecules have a tendency to form strongly quenching $\mathrm{H}$-aggregates at the outer surface of the ZL crystals (see, e.g., Figures 16 and 17 of ref 1 ). A new phenomenon was observed in the $\mathrm{Ox} 1^{+}$, perylene-ZL, as is illustrated in Figure 9. In this figure we compare the simulated $\left(\mathrm{A}^{\prime}\right)$ and measured $(\mathrm{A})$ emission and absorption spectra of $\mathrm{Ox}^{+}$, bone-DXP-ZL.3,.02; this is where $p$ $=0.3$ refers to the bone-DXP and 0.2 to the $\mathrm{Oxl}^{+}$. The simulated spectra were obtained by superimposing the spectra obtained from a bone-DXP-ZL.01 and an $\mathrm{Ox}^{+}-\mathrm{ZL} .01$ composite. The absorption and fluorescence spectra seen in Figure 9A stem from two different $\mathrm{Oxl}^{+}$, bone-DXP-ZL.3,02 samples. In one sample, care was taken to eliminate most of the $\mathrm{OxI}^{+}$adsorbed at the outer surface (red), and the washing procedure was less thorough in the other case (blue). Energy transfer from the bone-DXP to $\mathrm{OxI}^{+}$is in both cases clearly visible, as is the influence of self-absorption on the bone-DXP emission, causing a bathochromic shift and a change in its band shape. The $\mathrm{OxI}^{+}$fluorescence spectra of the two samples are, however, significantly different. Although the red curve matches well with the simulated spectrum, an important bathochromic shift is observed in the case of the blue curve. The emission band extends farther than $850 \mathrm{~nm}$, and the fluorescence quantum yield drops to about 0.06 compared to 0.26 in the case of the red curve. Samples with higher absorptivity reported in Table 2 have been prepared by simply adding more composites to the layer. Comparison with recent spectroscopic investigations of different $\mathrm{Oxl}^{+}$composites reported by Botta et al. allows the conclusion that the new band is caused by Jaggregate formation at the outer surface of the ZL host, probably close to the channel entrance. ${ }^{57,58}$ The latter point is a hypothesis that we cannot prove at the present time. A number of similar $\mathrm{Oxl}^{+}$, perylene-ZL composites have been prepared and analyzed, allowing us to say that the data shown in Figure $9 \mathrm{~A}$ is representative and can be regarded as typical. The same is true for the observation that the rapid decrease in fluorescence intensity in these samples matches the increase in absorptivity as seen in Table 2. This behavior can also be regarded as typical. The largest fluorescence yields we have measured in such samples are about $35 \%$. We conclude that preparing $\mathrm{Oxl}^{+}$,perylene-ZL composites with high luminescence quantum yields appears to be quite demanding. This can be mainly attributed to the difficulties in quantitatively suppressing aggregate formation and also to the speed of the energy migration in such a system. A faster energy migration massively increases the probability of reaching a quenching trap. Stable composites cannot be obtained without using a well-adapted channel-plugging procedure. The situation may be different by using the organization of type B shown in Scheme 1, where the
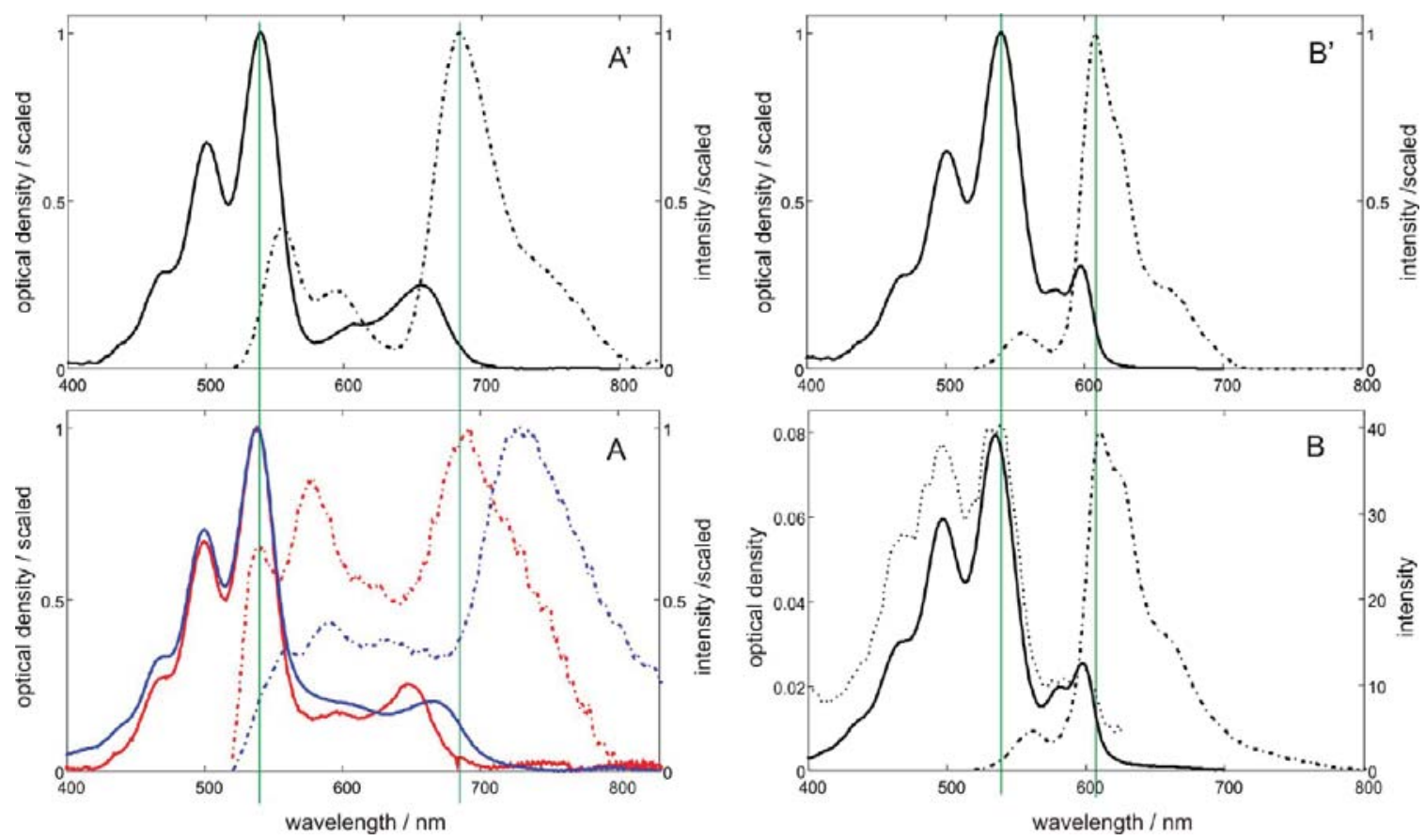

Figure 9. Absorption and emission spectra of donor,acceptor-ZL composites. (A, $\left.\mathrm{A}^{\prime}\right)$ Absorption (solid) and fluorescence (dash-dot) spectra of $\mathrm{Ox1}^{+}$,bone-DXP-ZL composites. (B,B') Similar spectra of tb-DXP,Ox ${ }^{+}-\mathrm{ZL}$ composites. All fluorescence spectra were excited at $490 \mathrm{~nm}$. $\left(\mathrm{A}^{\prime}\right)$ and $\left(\mathrm{B}^{\prime}\right)$ are simulated spectra obtained by superimposing absorption and fluorescence spectra of bone-DXP-ZL with Ox1 $1^{+}-\mathrm{ZL}$ or of tb-DXP-ZL with $\mathrm{Ox}^{+}-\mathrm{ZL}$ spectra, respectively. (A) Experimental spectra of two different samples of Ox1 ${ }^{+}$, bone-DXP-ZL.3,.02 composites scaled to the same height at their respective maxima. In one sample, care was taken to eliminate most of $\mathrm{Oxl}^{+}$adsorbed at the outer surface (red), but the washing procedure was less thorough in the other case (blue). (B) shows the absorption (solid), fluorescence (dash-dot) and excitation (dotted) spectra, detected at $675 \mathrm{~nm}$. The excitation spectrum was multiplied by a factor of 4.5 for better comparison. 
acceptors are inserted before the donors. We have realized this situation by first inserting $\mathrm{Ox}^{+}$into the channels by cation exchange from water. After washing and drying the material, tbDXP was added from the gas phase to obtain the tb-DXP, $\mathrm{Ox}^{+}-$ ZL.05,.3 composite. The perylene dyes are stabilized inside of the channels by means of interactions between the carbonyl oxygen with potassium cations, in a similar way as observed for fluorenone. ${ }^{46,47}$ Leaching is, therefore, much slower than for $\mathrm{Ox}^{+}$or $\mathrm{Ox}^{+}$. The perylene, $\mathrm{Ox}^{+}-\mathrm{ZL}$ composites are, therefore, much simpler to handle than $\mathrm{Ox}^{+}$, perylene-ZL. We compare in the right part of Figure 9 the simulated $\left(\mathrm{B}^{\prime}\right)$ and measured (B) spectra of tb-DXP, $\mathrm{Ox}^{+}-\mathrm{ZL} .05, .3$. The energy transfer from the tb-DXP donors to the $\mathrm{Ox}^{+}$acceptors is remarkable; $\mathrm{Ox}^{+}$ provides about $90 \%$ of the observed fluorescence intensity upon selective excitation of the donor at $490 \mathrm{~nm}$. This may be astonishing on the basis of the fact that the ETDM of $\mathrm{Ox}^{+}$is essentially oriented perpendicular to the channel axis, but this can be explained on the basis of the structure of the composite. ${ }^{2,51}$ The fluorescence quantum yield is large, about $70 \%$, indicating that many fewer traps are present because the channels are plugged with the tb-DXP and that self-absorption is much less important. We observe that the measured and the simulated spectra match very well. The experiments were extended by preparing composites with a donor to acceptor ratio of $45: 1$, namely tb-DXP, $\mathrm{Ox}^{+}-\mathrm{ZL} .004, .18$. These samples still showed almost quantitative energy transfer from the excited tb-DXP to the $\mathrm{Ox}^{+}$and a fluorescence quantum yield of about $90 \%$

\section{CONCLUSIONS}

Preparation and properties of dye-ZL and dye1,dye2-ZL composites are reported. All measurements have been carried out at ambient conditions, and most samples have been prepared using the $500 \mathrm{~nm}$ barrel-shaped ZL crystals. We were surprised to observe that the new tb-DXP, bone-DXP, and obone-DXP can be inserted into the ZL channels as easily as DXP, especially when the sterically demanding $t$-butyl group of tb-DXP is considered. Moreover, tb-DXP turned out to be especially convenient in this study due to its tendency to not show signs of J-coupling even at high loadings. Diluted tb$\mathrm{DXP}-\mathrm{ZL}$ is therefore better suited as a reference for relative fluorescence quantum yield measurements because the bulky $t$ butyl groups always keep the molecules at sufficient distance from each other inside of the ZL channels. Recording goodquality transmission spectra of the OGS layers was made possible because a background subtraction based on reference layers works well. The same is also true for polymer-embedded samples. This allows the comparison of spectra of diluted dyeZL composites with the corresponding spectra of diluted DCM solutions. We found that the influence of the host on the spectra is small, even astonishingly small and similar to what has recently been reported for methylacridine. ${ }^{15}$ This means that the reason for deviations seen in the spectra of dye-ZL composites from those measured in solution are of different origins. Intraparticle self-absorption and J-coupling are phenomena that depend on the loading of the sample. Jcoupling may also depend on the preparation conditions if the distribution equilibration of the dyes inside of the channels has not been fully established. Extraparticle self-absorption can be distinguished from the intraparticle process by diluting the OGS layers. The influence of self-absorption severely affects not only the shape of the fluorescence band but also the fluorescence quantum yield. Intraparticle self-absorption and re- emission cannot be avoided in dye-ZL composites of high loading both because of the large density of chromophores and also because of the pronounced optical anisotropy induced by the host. Of course, this effect also depends on the size of the ZL crystals, which is trivial and has, therefore, not been further discussed. Results of two differently organized light harvesting antenna of the dye1,dye2-ZL sandwich composite type with large amounts of donor with respect to acceptors present, as illustrated in Scheme 1, have been reported, and parameters influencing their properties have been discussed. These results allow a much better understanding of the different ways that can be used for preparing particles with desired absorption and fluorescence properties for photovoltaic and optoelectronic cells and other technologies using organic chromophores.

\section{AUTHOR INFORMATION}

\section{Corresponding Author}

*A. Devaux. E-mail: andre.devaux@unifr.ch.

\section{Author Contributions}

The manuscript was written through contributions by all authors. All authors have given approval to the final version of the manuscript.

\section{Notes}

The authors declare no competing financial interest.

Author Contact Information. A. Devaux, e-mail: andre.devaux@ unifr.ch. P. Belser, e-mail: peter.belser@unifr.ch. I. Miletto, email: ivana.miletto@unifr.ch. G. Calzaferri, e-mail: gion. calzaferri@iac.unibe.ch. O. Khorev, e-mail: oleg.khorev@ioc. unibe.ch. R. Häner, e-mail: robert.haener@ioc.unibe.ch. D. Brühwiler, e-mail: dominik.bruehwiler@zhaw.ch. A. Kunzmann, e-mail: andreas.kunzmann@optical-additives.com.

\section{ACKNOWLEDGMENTS}

Financial support by the Swiss Commission for Technology and Innovation (KTI/CTI, Project 12902.1 PFNM-NM) is gratefully acknowledged.

\section{REFERENCES}

(1) Calzaferri, G.; Huber, S.; Maas, H.; Minkowski, C. PhotonHarvesting Host-Guest Antenna Materials. Angew. Chem., Int. Ed. 2003, 42, 3732-3758.

(2) Calzaferri, G. Nanochannels: Hosts for Supramolecular Organization of Molecules and Complexes. Langmuir 2012, 28, 6216-6231.

(3) Mahato, R. N.; Lülf, H.; Siekman, M. H.; Kersten, S. P.; Bobbert, P. A.; de Jong, M. P.; De Cola, L.; van der Wiel, W. G. Ultrahigh Magnetoresistance at Room Temperature in Molecular Wires. Science 2013, 341, 257-260.

(4) Matsunaga, C.; Uchikoshi, T.; Suzuki, S.; Sakka, Y.; Matsuda, M. Fabrication of the c-Axis Oriented Zeolite L Compacts Using Strong Magnetic Field. Mater. Lett. 2103, 93, 408-410.

(5) Wen, T.; Zhang, W.; Hu, X.; He, L.; Li, H. Insight into the Luminescence Behavior of Europium(III) $\beta$-Diketonate Complexes Encapsulated in Zeolite L Crystals. ChemPlusChem 2013, 78, 438442.

(6) Li, Z.; Hüve, J.; Krampe, C.; Luppi, G.; Tsotsalas, M.; Klingauf, J.; De Cola, L.; Riehemann, K. Internalization Pathways of Anisotropic Disc-Shaped Zeolite L Nanocrystals with Different Surface Properties in HeLa Cancer Cells. Small 2013, 9, 1809-1820.

(7) Ban, T.; Takamura, M.; Morikawa, M.; Ohya, Y. Comparison of Organic Ligand Effects on Morphology Control Between Disc-Like Zeolite L Crystals and Zeolite A and W Crystals. Mater. Chem. Phys. 2013, 137, 1067-1072. 
(8) Szarpak-Jankowska, A.; Burgess, C.; De Cola, L.; Huskens, J. Cyclodextrin-modified Zeolites: Host-Guest Surface Chemistry for the Build-up of Multifunctional Nanocontainers. Chem.-Eur. J. 2013, DOI: $10.1002 /$ chem.201302153.

(9) Beierle, J. M.; Roswanda, R.; Erne, P. M.; Coleman, A. C.; Browne, W. R.; Feringa, B. L. An Improved Method for Site-Specific End Modification of Zeolite L for the Formation of Zeolite L and Gold Nanoparticle Self-Assembled Structures. Part. Part. Syst. Charact. 2013, 30, 273-279.

(10) Coutino-Gonzalez, E.; Roeffaers, M. B. J.; Dieu, B.; De Cremer, G.; Leyre, S.; Hanselaer, P.; Fyen, W.; Sels, B.; Hofkens, J. Determination and Optimization of the Luminescence External Quantum Efficiency of Silver-Clusters Zeolite Composites. J. Phys. Chem. C 2013, 117, 6998-7004.

(11) Manzano, H.; Gartzia-Rivero, L.; Bañuelos, J.; López-Arbeloa, I. Ultraviolet-Visible Dual Absorption by Single BODIPY Dye Confined in LTL Zeolite Nanochannels. J. Phys. Chem. C 2013, 117, 1333113336.

(12) Grüner, M.; Siozios, M. V.; Hagenhoff, B.; Breitenstein, D.; Strassert, C. A. Structural and Photosensitizing Features of Phthalocyanine-Zeolite Hybrid Nanomaterials. Photochem. Photobiol. 2013, DOI: $10.1111 /$ php.12141.

(13) Meeprasert, J.; Jungsuttiwong, S.; Namuangruk, S. Location and Acidity of Brønsted Acid Sites in Isomorphously Substituted LTL Zeolite: a Periodic Density Functional Study. Microporous Mesoporous Mater. 2013, 175, 99-106.

(14) Lim, H.; Choi, S.; Cheong, H.; Lee, J. S. Energy Transfer in Dye Molecule-Containing Zeolite Monolayers. Microporous Mesoporous Mater. 2013, DOI: 10.1016/j.micromeso.2013.08.012.

(15) Fois, E.; Tabacchi, G.; Devaux, A.; Belser, P.; Brühwiler, D.; Calzaferri, G. Host-Guest Interactions and Orientation of Dyes in the One-Dimensional Channels of Zeolite L. Langmuir 2013, 29, 91889198.

(16) Lupulescu, A. I.; Kumar, M.; Rimer, J. D. A Facile Strategy To Design Zeolite L Crystals with Tunable Morphology and Surface Architecture. J. Am. Chem. Soc. 2013, 135, 6608-6617.

(17) Pauchard, M.; Huber, S.; Méallet-Renault, R.; Maas, H.; Pansu, R.; Calzaferri, G. Time and Space Resolved Luminescence of a New Dye-Zeolite Photonic Antenna. Angew. Chem., Int. Ed. 2001, 40, 2839-2842.

(18) Megelski, S.; Lieb, A.; Pauchard, M.; Drechsler, A.; Glaus, S.; Debus, C.; Meixner, A. J.; Calzaferri, G. Orientation of Fluorescent Dyes in the Nano Channels of Zeolite L. J. Phys. Chem. B 2001, 105, $25-35$.

(19) Gasecka, A.; Dieu, L.-Q.; Brühwiler, D.; Brasselet, S. Probing Molecular Order in Zeolite L Inclusion Compounds Using TwoPhoton Fluorescence Polarimetric Microscopy. J. Phys. Chem. B 2010, 114, 4192-4198.

(20) Calzaferri, G.; Méallet-Renault, R.; Brühwiler, D.; Pansu, R.; Dolamic, I.; Dienel, T.; Adler, P.; Li, H.; Kunzmann, A. Designing Dye-Nanochannel Antenna Hybrid Materials for Light Harvesting, Transport and Trapping. ChemPhysChem 2011, 12, 580-594.

(21) Busby, M.; Devaux, A.; Blum, Ch.; Subramaniam, V.; Calzaferri, G.; De Cola, L. Interactions of Perylene Bisimide in the OneDimensional Channels of Zeolite. J. Phys. Chem. C 2011, 115, 59745988.

(22) Hashimoto, S. Optical Spectroscopy and Microscopy Studies on the Spatial Distribution and Reaction Dynamics in Zeolites. J. Phys. Chem. Lett. 2011, 2, 509-519.

(23) Gartzia-Rivero, L.; Bannuelos-Prieto, J.; Martinez-Martinez, V.; Lopez Arbeloa, I. Versatile Photoactive Materials Based on Zeolite L Doped with Laser Dyes. ChemPlusChem 2012, 77, 61-70.

(24) Nicolet, O.; Huber, S.; Lovey, C.; Chappellet, S.; Perrenoud, J.; Pauchard, M.; Ferrini, R.; Zuppiroli, L. Quantum Yield Measurement of Fluorescent Zeolite Nanopigments. Adv. Funct. Mater. 2009, 19, 17.

(25) Devaux, A.; Popovic, Z.; Bossart, O.; De Cola, L.; Kunzmann, A.; Calzaferri, G. Solubilization of Dye-Loaded Zeolite L Nanocrystals. Microporous Mesoporous Mater. 2006, 90, 69-72.
(26) Suárez, S.; Devaux, A.; Bañnuelos, J.; Bossart, O.; Kunzmann, A.; Calzaferri, G. Transparent Zeolite-Polymer Hybrid Materials with Tunable Properties. Adv. Func. Mater. 2007, 17, 2298-2306.

(27) Calzaferri, G.; Lutkouskaya, K. Mimicking the Antenna System of Green Plants. Photochem. Photobiol. Sci. 2008, 7, 879-910.

(28) Baerlocher, Ch.; McCusker, L. B.; Olson, D. H. Atlas of Zeolite Framework Types, 6th ed.; Elsevier: Amsterdam, 2007.

(29) Ohsuna, T.; Slater, B.; Gao, F.; Yu, J.; Sakamoto, Y.; Zhu, G.; Terasaki, O.; Vaughan, D. E.; Qiu, S.; Catlow, C. R. Fine Structures of Zeolite-Linde-L (LTL): Surface Structures, Growth Unit and Defects. Chem.-Eur. J. 2004, 10, 5031-5040.

(30) Maas, H.; Calzaferri, G. Trapping Energy from and Injecting Energy into Dye-Zeolite Nanoantennae. Angew. Chem., Int. Ed. 2002, $41,2284-2288$

(31) Calzaferri, G.; Huber, S.; Maas, H.; Minkowski, C. PhotonHarvesting Host-Guest Antenna Materials. Angew. Chem., Int. Ed. 2003, 42, 3732-3758.

(32) Bossart, O.; De Cola, L.; Welter, S.; Calzaferri, G. Injecting Electronic Excitation Energy Into an Artificial Antenna System through a $\mathrm{Ru}^{2+}$ Complex. Chem.-Eur. J. 2004, 10, 5771-5775.

(33) Ban, T.; Brühwiler, D.; Calzaferri, G. Selective Modification of the Channel Entrances of Zeolite L with Triethoxysilylated Coumarin. J. Phys. Chem. B 2004, 108, 16348-16352.

(34) Tsotsalas, M.; Kopka, K.; Luppi, G.; Wagner, S.; Law, M. P.; Schäfers, M.; De Cola, L. Encapsulating ${ }^{111}$ In in Nanocontainers for Scintigraphic Imaging: Synthesis, Characterization, and In Vivo Biodistribution. ACS Nano 2010, 4, 342-348.

(35) Kehr, N. S.; Riehemann, K.; El-Gindi, J.; Schäfer, A.; Fuchs, H.; Galla, H.-J.; De Cola, L. Cell Adhesion and Cellular Patterning on a Self-Assembled Monolayer of Zeolite L Crystals. Adv. Funct. Mater. 2010, 20, 2248-2254.

(36) Calzaferri, G.; Devaux, A. Manipulation of Energy Transfer Processes within the Channels of L-Zeolite. in Supramolecular Photochemistry: Controlling Photochemical Processes; Ramamurthy, V., Inoue, Y. Eds.; John Wiley \& Sons: Hoboken, NJ, 2011; 285-387.

(37) López-Duarte, I.; Dieu, L.-Q.; Dolamic, I.; Martínez-Díaz, M. V.; Torres, T.; Calzaferri, G.; Brühwiler, D. On the Significance of the Anchoring Group in the Design of Antenna Materials Based on Phthalocyanine Stopcocks and Zeolite L. Chem.-Eur. J. 2011, 17, $1855-1862$

(38) El-Gindi, J.; Benson, K.; De Cola, L.; Galla, H.-J.; Kehr, N. S. Cell Adhesion Behavior on Enantiomerically Functionalized Zeolite L Monolayers. Angew. Chem., Int. Ed. 2012, 51, 3716 -3720.

(39) Pauchard, M.; Devaux, A.; Calzaferri, G. Dye-Loaded Zeolite L Sandwiches as Artificial Antenna Systems for Light Transport. Chem., Eur. J. 2000, 6, 3456-3470.

(40) Maas, H.; Khatyr, A.; Calzaferri, G. Phenoxazine Dyes in Zeolite L, Synthesis and Properties. Microporous Mesoporous Mater. 2003, 65, 233-242.

(41) Rademacher, A.; Märkle, S.; Langhals, H. Lösliche PerylenFluoreszenzfarbstoffe mit hoher Photostabilität. Chem. Ber. 1982, 115, 2927-2934.

(42) Zabala Ruiz, A.; Brühwiler, D.; Ban, T.; Calzaferri, G. Synthesis of Zeolite L: Tuning Size and Morphology. Monatsh. Chem. 2005, 136, 77-89.

(43) Gaona Gomez, A.; de Silveira, G.; Doan, H.; Cheng, C.-H. A Facile Method to Tune Zeolite L Crystals with Low Aspect Ratio. Chem. Commun. 2011, 47, 5876-5878.

(44) Lakowicz, J. R. Principles of Fluorescence Spectroscopy, 3rd ed.; Springer: Singapore, 2006.

(45) El-Daly, S. A. Spectral, Lifetime, Fluorescence Quenching, Energy Transfer and Photodecomposition of N,N-bis(2,6-dimethyl phenyl)-3,4:9,10-perylentetracarboxylic Diimide (DXP). Spectrochim. Acta, Part A 1999, 55, 143-152.

(46) Fois, E.; Tabacchi, G.; Calzaferri, G. Interactions, Behavior and Stability of Fluorenone inside Zeolite Nanochannels. J. Phys. Chem. C 2010, 114, 10572-10579. 
(47) Devaux, A.; Minkowski, C.; Calzaferri, G. Electronic and Vibrational Properties of Fluorenone in the Channels of Zeolite L. Chem.-Eur. J. 2004, 10, 2391-2408.

(48) Calzaferri, G.; Brühwiler, D.; Meng, T.; Dieu, L.-Q.; Malinovskii, V. L.; Häner, R. Surprising Properties of a Furo-Furanone. Chem.Eur. J. 2010, 16, 11289-11299.

(49) Landgraf, S.; Grampp, G. A Subnanosecond Time-Resolved Fluorescence Lifetime Spectrometer Applying Laser Diodes. Monatsh. Chem. 2000, 131, 839-848.

(50) Calzaferri, G.; Gfeller, N. Thionine in the Cage of Zeolite L. J. Phys. Chem. 1992, 96, 3428-3435.

(51) Fois, E.; Tabacchi, G.; Calzaferri, G. Orientation and Order of Xanthene Dyes in the One-Dimensional Channels of Zeolite L: Bridging the Gap between Experimental Data and Molecular Behavior. J. Phy. Chem. C 2012, 116, 16748-16799.

(52) Hammond, P. R. Self-Absorption of Molecular Fluorescence, the Design of Equipment for Measurement of Fluorescence Decay, and the Decay Times of some Laser Dyes. J. Chem. Phys. 1979, 70, 3884-3894.

(53) Calzaferri, G.; Brühwiler, D.; Megelski, S.; Pfenniger, M.; Pauchard, M.; Hennessy, B.; Maas, H.; Devaux, A.; Graf, U. Playing with Dye Molecules at the Inner and Outer Surface of Zeolite L. Solid State Sci. 2000, 2, 421-447.

(54) Batchelder, J. S.; Zewail, A. H.; Cole, T. Luminescent Solar Concentrators. 1: Theory of Operation and Techniques for Performance Evaluation. Appl. Opt. 1979, 18, 3090-3110.

(55) Yatskou, M.; Pfenniger, M.; Meyer, M.; Huber, S.; Calzaferri, G. Electronic Excitation Energy Migration in a Photonic Dye-Zeolite Antenna. ChemPhysChem 2003, 4, 567-587.

(56) Albuquerque, R. Q.; Calzaferri, G. Proton Activity Inside the Channels of Zeolite L. Chem.-Eur. J. 2007, 13, 8939-8952.

(57) Leone, G.; Giovanella, U.; Porzio, W.; Botta, Ch.; Rizzi, G. In Situ Synthesis of Fluorescent Poly(norbornene)/Oxazine-1 Dye Loaded Fluoromica Hybrids: Supramolecular Control over Dye Arrangement. J. Mater. Chem. 2011, 21, 12901-12909.

(58) Giovanella, U.; Leone, G.; Ricci, G.; Virgili, T.; Lopez, I. S.; Rajendran, S. K.; Botta, Ch. Oxazine-1 J-Aggregates in Polymer Nanohybrids. Phys. Chem. Chem. Phys. 2012, 14, 13646-13650. 